NOTICE WARNING CONCERNING COPYRIGHT RESTRICTIONS:

The copyright law of the United States (title 17, U.S. Code) governs the making of photocopies or other reproductions of copyrighted material. Any copying of this document without permission of its author may be prohibited by law. 


\title{
A UNIVERSAL DOMAIN TECHNIQUE FOR PROFINITE POSETS ${ }^{1}$
}

\author{
by \\ Carl A. Gunter ${ }^{2}$ \\ Department of Computer Science \\ Carnegie-Mellon University \\ Pittsburgh, Pennsylvania 15213 \\ July 3,1985
}

\begin{abstract}
We introduce a category of what we call profinite domains. This category of domains differs from most of the familiar oncs in having a categorical coproduct as well as being cartesian closed. Study of these domains is carried out through the use of an equivalent category of pre-orders in a manner similar to the information systems approach advocated by Dana Scott and others. A class of universal profinite domains is defined and used to derive sufficient conditions for the profinite solution of domain cquations involving continuous operators. Necessary conditions for the existence of such solutions are also given and used to derive results about solutions of some important equations. A new universal bounded complete domain is also demonstrated using an operator which has bounded complete domains as its fixed points.
\end{abstract}

\section{INTRODUCTION.}

For our purposes a domain equation has the form $X \cong F(X)$ where $F$ is an operator on a class of semantic domains (typically, $F$ is an cndofunctor on a category $\mathbf{C}$ ). The solution of such equations is a major component of the Scott-Strachey approach to programming language semantics. The reader is refered to [23] or any other reference on denotational semantics for an explanation of how such equations arise. Techniques for solving them have been worked out for specific categories (see any of the references by Scull or Plotkin) and in rather general categorytheoretic settings as well [22]. Computability considerations have been incorporated into many of these treatments with considerable success $([24],[5],[6])$. All of these approaches use one of three techniques. The most general is the inverse limit construction used by Scott [14] to solve the domain equation $D \cong[D \rightarrow D]$ (where $[D \rightarrow D]$ is the function space of $D$ ). The second uses the Tarski Fixed Point Theorem, which says: if $D$ is a poset with least upper bounds for $\omega$-chains and a

\footnotetext{
${ }^{1}$ A preliminary version of this paper appeared in the proceedings of ICALP 1985 .

${ }^{2}$ This research was sponsored by the Defense Advanced Research Projects Agency (DOD), ARPA Order No. 3507, monitored by the Air Force Avionics Laboratory under Contract F33615-84-K-1520. The views and conclusions contained in this document are those of the author and should not be interpreted as representing the official policies, either expressed or inplicd, of the Defense Advanced Researcl Projects Agency or the US Goverument.
} 
least element then any function $f: D \hookrightarrow D$ which preserves such lub's has a least fixed point. The third-which is introduced in [9]-uses the Banach Fixed Point Theorem, which says: a uniformly contractive function $f: X \rightarrow X$ on a non-cmpty complete metric spacc $X$ has a unique fixed point. These last two approaches employ what is frequently called a "universal" domain to associate with the operator $F$ a lub preserving or contractive map.

In this paper we examine the problems involved in obtaining solutions to equations over the category of profinite domains which will be defined below. This is a rather natural, and in a sense inevitable, category which contains SFP (sec [11]) as a full subcategory. It has the unusual property of being bicartesian closed, i.e. it is cartesian closed and has a coproduct. Such categories have a rich type structure and form models of the typed $\lambda$-calculus [8]. Obtaining profinite solutions for domain equations involving the coproduct can be problematic, however. For example, the equation $D \cong 1+[D \rightarrow D]$ where 1 is the one point domain has no profinite solution. We provide a necessary condition which, in effect, reduces the problem of solving such an equation over the profinite domains to one of getting a finite poset which solves a related equation. This condition is proved sufficient by a variant of the second method described above. Since no single (projection) universal domain for the profinites exists we derive an infinite class of domains which are "sufficiently universal" to apply the method. As a secondary theme we show how to extend the neighborhood or information system approach to one which applies to categories (such as SFP) which are larger than the one considered in [16] and [18]. We also illustrate a method of solving domain equations up to equality similar to the one demonstrated in [18] and [24] by using such a technique to solve a domain equation involving the convex powerdomain.

Section two gives some of the basic definitions and explains the equivalence defined by the ideal completion functor. In section three the category of Plotkin orders is introduced and shown to be bicartesian closed. Section four discusses normal substructures and defines the category of profinite domains. We also state a result which shows that this category is "maximal" with respect to the conditions of $\omega$-algebraicity and cartesian closure. Section five derives universal domains. In section six an interesting operator which we call the join completion is discussed. In section seven the universal domains arc uscd to show existence of solutions for a significant class of equations. Section seven also contains discussion of several specific domain equations and their solutions.

\section{PRE-ORDERS AND ALGEBRAIC DCPO'S.}

A pre-order is a pair $\left\langle A, \vdash_{A}\right\rangle$ where $\vdash_{A}$ is a binary relation which is reflexive and transitive. It is intended that the "larger" element is the one on the left side of the turnstile. Note that $A=\emptyset$ is allowed. To conserve notation we write $A=\left\langle A, \vdash_{A}\right\rangle$ and when $A$ is clear from context the subscript is dropped. A set $S \subseteq A$ is bounded if there is an $X \in A$ such that $X \vdash Y$ for every $Y \in S$. Such an $X$ is called a bound for $S$ and we write $X \vdash S$. Trivially, any $X \in A$ is a bound for the empty set. A subset $M \subseteq A$ of a pre-order $A$ is directed if every finite subset of $M$ has a bound in $M$. 
Note, in particular, that every directed set is non-empty. The following definition appears in [12] and [19].

Definition: An approximable relation $f: A \rightarrow B$ is a subset of $A \times B$ which satisfies the following axioms for any $X, X^{\prime} \in A$ and $Y, Y^{\prime} \in B$ :

1. for every $X \in A$ there is a $Y \in A$ such that $X f Y$;

2. if $X f Y$ and $X f Y^{\prime}$ then there is a $Z \in B$ such that $X f Z$ and $Z \vdash_{B} Y, Y^{\prime}$;

3. if $X \vdash_{A} X^{\prime} f Y^{\prime} \vdash_{B} Y$ then $X f Y$.

Let $g: A \rightarrow B$ and $f: B \rightarrow C$ be approximable relations. We define a binary relation $f \circ g$ on $A \times C$ as follows. For each $X \in A$ and $Z \in C, X(f \circ g) Z$ if and only if there is a $Y \in B$ such that $X g Y$ and $Y f Z$. Also, for each pre-order $A$ define id ${ }_{A}=\vdash_{A}$. It is easy to verify that $f \circ g$ and $\mathrm{id}_{A}$ are approximable relations. With this composition and identity relation the class of pre-orders and approximable relations form a category which we call $\mathrm{PO}$. We let $\mathbf{P O}(A, B)$ be the set of approximable relations on $A \times B$. The approximable relations are partially ordered by set theoretic inclusion.

For pre-orders $A$ and $B$ define the product pre-order to have the coordinatewise ordering,

$$
(X, Y) \vdash_{A \times B}\left(X^{\prime}, Y^{\prime}\right) \text { if and only if } X \vdash_{A} X^{\prime} \text { and } Y \vdash_{B} Y^{\prime}
$$

In fact, $x$ is a categorical product for PO. If we take 1 to be the single element prc-order, then for each pre-order $A$ there is a unique approximable relation $1_{A}: A \rightarrow 1$. Thus the pre-orders and approximable relations form a cartesian category with terminal object 1 . Moreover, the empty poset 0 , is initial in this category, i.e. for any object $A$ there is a unique arrow $0_{A}: 0 \rightarrow A$. This $0_{A}$ is the "empty relation" and it is trivially approximable. For pre-orders $A$ and $B$, the coproduct pre-order $\left\langle A+B, \vdash_{A+B}\right\rangle$ is defined by letting $A+B=(A \times\{0\}) \cup(B \times\{1\})$ and defining $(X, n) \vdash_{A+B}(Y ; m)$ if and only if either

1. $n=m=0$ and $X \vdash_{A} Y$, or

2. $n=m=1$ and $X \vdash_{B} Y$.

This, unlike the + operators discussed in many references on domain theory such as [23], [2], or [18] is the categorical coproduct in PO. This shows that PO is bicartesian, i.e. it has coproduct and initial object as well as product and terminal object.

A poset $\langle D, \sqsubseteq\rangle$ (or partially ordered set) is a pre-order that is anti-symmetric, i.e. if $x \sqsubseteq y$ and $y \sqsubseteq x$ then $x=y$. Using the established convention we write the "larger" element on the right side of the $\sqsubseteq$ symbol. If $x \sqsubseteq y$ then it is sometimes convenient to write $y \sqsupseteq x$. If $x \sqsubseteq y$ and $x \neq y$ then we write $x \sqsubset y$; we define $\sqsupset$ by a similar convention. It is frequently desirable to transfer a property of pre-orders to a property of posets and conversely. This is usually possible because pre-orders and posets are closely connected. First of all, every pre-order is isomorphic (in the category with approximable relations as arrows) to a poset. To see this, let $\langle A, H\rangle$ be a pre-order. Define an equivalence relation $\sim$ on $A$ by letting $X \sim Y$ if and only if $X \vdash Y$ and $Y \vdash X$. For each $X$, let 
$\tilde{X}=\{Y \in A \mid X \sim Y\}$ and set $\tilde{A}=\{\tilde{X} \mid X \in A\}$. If we define a binary relation $\sqsubseteq$ on $A$ by letting $\tilde{Y} \sqsubseteq \tilde{X}$ if and only if $X \vdash Y$, then it is easy to check that $\langle\tilde{A}, \sqsubseteq\rangle$ is a poset and the approximable relation $f: \tilde{A} \rightarrow A$ given by $\tilde{X} f Y$ if and only if $X \vdash Y$ is an isomorphism. In addition, posets are isomorphic in the category with approximable relations as arrows if and only if they are isomorphic in the more familiar category with monotone maps as arrows. We can therefore write $A \cong B$ for pre-orders $A$ and $B$ without fear of ambiguity.

A poset $\langle D, \sqsubseteq\rangle$ is said to be a directed complete if every directed subset $M \subseteq D$ has a least upper bound $\bigsqcup M \in D$. A monotone function $f: D \rightarrow E$ between dcpo's $D$ and $E$ is continuous if for every directed set $M \subseteq D, \bigsqcup f(M)=f(\bigsqcup M)$. The dcpo's and continuous functions form a category; with a slight over-loading of notation we let $\mathrm{id}_{D}: D \rightarrow D$ denote the identity function. Let $[D \rightarrow E]$ be the set of continuous functions from $D$ to $E$. We order $[D \rightarrow E]$ by setting $f \subseteq g$ if for every $x \in D, f(x) \sqsubseteq g(x)$. It is easy to check that $[D \rightarrow E]$ is itself a depo. This definition of dcpo's differs from most other definitions in the literature. We do not require that a depo have a least element; indeed, we do not require a dcpo to be non-empty. Much of the usual theory of dcpo's goes through for these "bottomless" cases but there are some non-trivial differences. For example, a continuous function $f: D \rightarrow D$ on a dcpo need not have a fixed point. (However, if for some $x \in D, x \sqsubseteq f(x)$ then there is a least $y \sqsupseteq x$ such that $f(y)=y$.)

Let $D$ be a depo. An element $x \in D$ is finite (or compact) if whenever $x \sqsubseteq \bigsqcup M$ for a directed set $M$, there is a $y \in M$ such that $x \sqsubseteq y$. Let $\mathbf{B}[D]$ denote the set of finite elements of a depo $D$. We say that $D$ is algebraic if for every $x \in D$, the set $M=\left\{x_{0} \in \mathbf{B}[D] \mid x_{0} \sqsubseteq x\right\}$ is directed and $\cup M=x$. In other words, in an algebraic dcpo every element is the limit of its finite approximations. Let AIG be the category of algebraic dcpo's and continuous functions. We now establish an equivalence between ALG and PO. Suppose $\langle A, \vdash\rangle$ be a pre-order. An ideal over $\mathbf{A}$ is a directed subset $x \subseteq A$ such that if $X \vdash Y$ and $X \in x$ then $Y \in x$. The ideal completion of $A$ is the partial ordering, $\langle|A|, \subseteq\rangle$, of the ideals of $A$ by set-theoretic inclusion. If $X \in A$ then the principal ideal generated by $X$ is the set $\downarrow X=\{Y \in A \mid X \vdash Y\}$. Note that $\{\downarrow X \mid X \in A\} \cong A$. We also have the following:

Theorcm 1: If $A$ is a pre-order, then $|A|$ is an algebraic dcpo with $\mathbf{B}\|A\|=\{|X| X \in A\}$. Moreover, every algebraic dcpo $D$ is representable in this way because $D \cong|\mathbf{B}[D]|$.

Proof. Note that if $M \subseteq|A|$ is directed then $\bigcup M$ is the least upper bound of $M$. Hence $|A|$ is a dcpo. If $X \in A$ and $\downarrow X \subseteq \cup M$ then $X \in y$ for some $y \in M$ so $\downarrow X \subseteq y$. Hence $\downarrow X$ is finite (as an element of $|A|$ ). But for any ideal $x$, the set $M=\{\downarrow X \mid X \in x\}$ is dirccted (because $x$ is directed) and $x=\bigcup M$. Hence $|A|$ is an algebraic dcpo and $\mathbf{B} \| A \mid\}=\{\downarrow X \mid X \in A\}$ is isomorphic to $A$. On the other hand, if $\langle D, \sqsubseteq\rangle$ is an algebraic dcpo then it is easy to verify that $f: D \rightarrow|\mathbf{B}[D]|$ by $f(x)=\left\{x_{0} \mid x_{0} \sqsubseteq x\right\}$ is an isomorphism.

Intuitively, the passage $A \mapsto|A|$ expands $A$ by adding limits for ascending chains. To see this in a specific example, let $<\omega_{2}$ be the set of functions $f: n \rightarrow 2$ where $n<\omega$. If $f: n \rightarrow 2$ and $g: m \rightarrow 2$ then say $f \sqsubseteq g$ if and only if $n<m$ and $f(k)=g(k)$ for each $k<n$. the ideal 
completion $\left.\right|^{<\omega} 2 \mid$ of this poset is isomorphic to the union ${ }^{<\omega} 2 \cup^{\omega} 2$ where ${ }^{\omega} 2$ is the set of functions from $\omega$ into 2 ,

- ${ }^{<\omega} 2$ retains the ordering just mentioned and

- if $f: n \rightarrow 2$ and $g: \omega \rightarrow 2$ then $f \sqsubseteq g$ if and only if $f(k)=g(k)$ for each $k<n$.

The infinite elements of $\left.\right|^{<\omega} 2 \mid$ correspond to those in ${ }^{\omega} 2$ while the finite elements of $\left.\right|^{<\omega} 2 \mid$ correspond to those in $<\omega^{\omega}$. If a poset $A$ has no infinite chains then surely no new elements are added by the ideal completion. We make this intuition precise as follows.

Definition: A poset $\langle A, \sqsubseteq\rangle$ is said to have the ascending chain condition (acc) if for every chain $X_{0} \sqsubseteq X_{1} \sqsubseteq X_{2} \sqsubseteq \cdots$ of elements of $A$ there is an $n \in \omega$ such that for every $m \geq n, X_{m}=X_{n}$. A pre-order $\langle A, \vdash\rangle$ is said to have the acc if $\tilde{A}$ does.

Proposition 2: If $\langle A, \vdash\rangle$ has the acc then $A \cong|A|$.

Proof. We show below that $|A| \cong|B|$ if $A \cong B$. Since $A \cong \tilde{A}$ we can therefore assume that $A$ is a poset. We show that each $x \in|A|$ is principal. Assume $x \in|A|$ is not principal. Then for each $X \in x$ there is an $X^{\prime} \in x$ such that $X \sqsubset X^{\prime}$. But this means there is a chain $X_{0} \sqsubset X_{1} \sqsubset \cdots$ of elements of $x$. This contradicts the assumption that $A$ has the acc. Hence $|A|=\{\downarrow X \mid X \in A\} \cong A$.

口

A rather obvious corollary of the Proposition is that all finite posets are algebraic depo's. Now, if $D$ is a poset with the acc and $M \subseteq D$ is directed then $\bigsqcup M=x$ for some $x \in M$. Hence, if $f: D \rightarrow E$ is monotone then $f(\sqcup M)=f(x)=\sqcup f(M)$. We conclude that when $D$ has the acc then $[D \rightarrow E]$ is just the set of monotone functions from $D$ into $E$.

There is a sense in which $|A|$ is freely generated by $A$. Formally, wc have the following:

Theorem 3: Let $A$ be a pre-order and suppose $\phi: A \rightarrow|A|$ by $\phi: X \mapsto \downarrow X$. Then for every dcpo $D$ and monotone function $f$ there is a unique continuous function $\vec{f}$ such that the following diagram commutes.

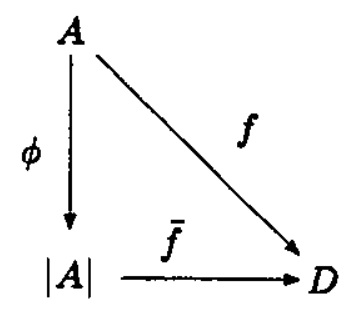

Moreover, the correspondence $f \mapsto \bar{f}$ is monotone.

Proof. Let $f$ and $D$ be given as in the theorem. Define $\vec{f}$ by

$$
\bar{f}(x)=\sqcup\{f(X) \mid X \in x\} .
$$


This makes sense because $f$ is monotone, $x$ is directed and $D$ is complete. To sce that $\bar{f}$ is continuous, suppose $M$ is a dirccted subset of $|A|$. Then

$$
\begin{aligned}
\vec{f}(\bigcup M) & =\bigsqcup\{f(X) \mid X \in \bigcup M\} \\
& =\bigsqcup\{f(X) \mid X \in x \text { for some } x \in M\} \\
& =\bigsqcup\{\bigsqcup\{f(X) \mid X \in x\} \mid x \in M\} \\
& =\bigsqcup \bar{f}(M) .
\end{aligned}
$$

To sce that $\bar{f}$ is unique, suppose $g:|A| \rightarrow D$ is continuous and for every $X \in A, g(\downarrow X)=f(X)$. Then

$$
\begin{aligned}
g(x) & =\bigsqcup\{g(\downarrow X) \mid X \in x\} \\
& =\bigsqcup\{f(X) \mid X \in x\} \\
& =\bar{f}(x) .
\end{aligned}
$$

That the function $f \mapsto \bar{f}$ is monotone is easy to check given the above definition of $\bar{f}$.

Definition: If $A$ and $B$ are pre-orders and $f: A \rightarrow B$ is an approximable relation then define a function $|f|:|A| \rightarrow|B|$ by $|f|(x)=\{Y \mid X f Y$ for some $X \in x\}$.

Note that the conditions set down in the definition of an approximable relation insure that the set on the right actually is in $|B|$.

Theorem 4: Let $A$ and $B$ be pre-orders. If $f: A \rightarrow B$ is approximable then $|f|:|A| \rightarrow|B|$ is continuous. Moreover, the correspondence $f \mapsto|f|$ is an isomorphism between the posets $\mathrm{PO}(A, B)$ and $\| A|\rightarrow| B \mid]$.

Proof. To see that $|f|$ is continuous, suppose $M \subseteq|A|$ is directed. Then

$$
\begin{aligned}
\bigcup|f|(M) & =\bigcup\{|f|(x) \mid x \in M\} \\
& =\bigcup\{\{Y \mid X f Y \text { for some } X \in x\} \mid x \in M\} \\
& =\{Y \mid X f Y \text { for some } X \in U M\} \\
& =|f|(\bigcup M) .
\end{aligned}
$$

Now, suppose $f:|A| \rightarrow|B|$ is continuous. Define a relation $\langle f\rangle \subseteq A \times B$ by letting $X\langle f\rangle Y$ if and only if $Y \in f(\downarrow X)$. For any $x \in|A|$ we have

$$
\begin{aligned}
|\langle f\rangle|(x) & =\{Y \mid X\langle f\rangle Y \text { for some } X \in x\} \\
& =\{Y \mid Y \in f(\downarrow X) \text { for some } X \in x\} \\
& =\bigcup\{f(\downarrow X) \mid X \in x\} \\
& =f(x)
\end{aligned}
$$

since $f$ is continuous. On the other hand, if $f \subseteq A \times B$ is approximable then $X\langle|f|\rangle Y$ if and only if $Y \in|f|(\downarrow X)$ if and only if $X f Y$. Hence $\langle|f|\rangle=f$. Now, if $f \subseteq g$ for approximable relations $f$ and $g$ then

$$
\begin{aligned}
|f|(x) & =\{Y \mid X f Y \text { for some } X \in x\} \\
& \subseteq\{Y \mid X g Y \text { for some } X \in x\} \\
& =|g|(x)
\end{aligned}
$$


On the other liand, suppose $\int . g:|B| \rightarrow|A|$ are continuous. If $\int E g$ and $X\langle f\rangle Y$ then $Y \in$ $f(\downarrow X) \subseteq g(\downarrow X)$ so $X\langle g\rangle Y$. Hence $\langle f\rangle \subseteq\langle g\rangle$. Wo conchude that $\mathbf{P O}(A, B) \cong\|A|\rightarrow| B\|$.

Suppose that $g: A \rightarrow B$ and $f: B^{\circ} \rightarrow C$ are approximable relations. Then for any $x \in|A|$. one can show that $(|f| \circ|g|)(x)=|f \circ g|(x)$. Since $\left|i_{A}\right|(x)=x$ for any proorder $A$ and $x \in|A|$ we may conclude that the passage $A \mapsto|A|, f \mapsto|f|$ is a functor. In category theoretic terminology, Theorem 1 says that this functor is dense and Theorem 4 says that it is full and faithful. Wo have therefore proved the following:

Theorem 5: The category of pre-orders and approximable relations is equivalent (in the category theoretic sense) to the category of algebraic dcpo's.

This equivalence extends to several interesting subcategories as well. If $K$ is a class of pre-orders then let $\operatorname{Id}_{K}$ be the category which has as objects algebraic depo's $D$ such that $\mathbf{B}[D]$ is isomorphic to a pre-order in $K$ and has as arrows continuous functions. If $K$ is the class of upper semi-lattices then $I d_{K}$ is the category of algebraic lattices. Let us say that a pre-order $A$ is coherent if whenever a finite $u \subseteq A$ is pair-wise bounded then it has a least upper bound. If $K$ is the class of coherent pre-orders then it is possible to show that $\mathbf{I d} \mathbf{d}_{\mathcal{K}}$ is the category of coherent algebraic dcpo's. A (non-empty) pre-order is bounded complete if each of its finite bounded subsets has a least upper bound. Again, if $K$ is the class of bounded complete pre-orders then it is possible to show that Id $\mathbf{d}_{K}$ is the category of bounded complete dcpo's. Each of these three categories is cartesian closed but none of them has a categorical coproduct. Note also that there is an equivalence between the category of countable pre-orders and the category of countably based algebraic dcpo's.

\section{PLOTKIN ORDERS.}

In this section we introduce the category of Plotkin orders which will be our primary technical tool for studying the profinite domains. Plotkin orders are less abstract than profinite domains and in many ways they are easier to work with. For example, Smyth [21] proves many facts about strongly algebraic domains by taking a detailed look at the particular class of Plotkin orders which "correspond" to such domains. Their use makes some arguments more algebraic and simplifies the definitions of some of the operators such as the powerdomains which we discuss later.

Definition: Suppose $A$ is a pre-order and $S \subseteq A$. We say that $S$ is normal in $A$ and write $S \triangleleft A$ if for every $X \in A$ the set $S \cap \downarrow X$ is directed.

Note, incidently, that if $S \triangleleft A$ and $X \in A$ then $X \vdash \emptyset$ and $\emptyset \subseteq S$, so there is an $X^{\prime} \in S$ such that $X \vdash X^{\prime}$. Let $u$ be a subset of $A$. A set $u^{\prime}$ of upper bounds of $u$ is said to be complete if whenever $X \vdash u$, there is an $X^{\prime} \in u^{\prime}$ such that $X \vdash X^{\prime}$. We summarize some more of the properties of the $\triangleleft$ relation in the following

Lemma 6: Let $A, B, C$ be pre-orders.

1. Suppose $A \subseteq B$. Then $A \triangleleft B$ if and only if for every $u \subseteq A$ there is a set $u^{\prime} \subseteq A$ of upper 


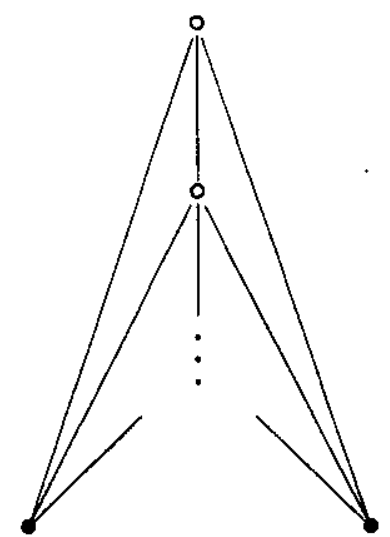

a.

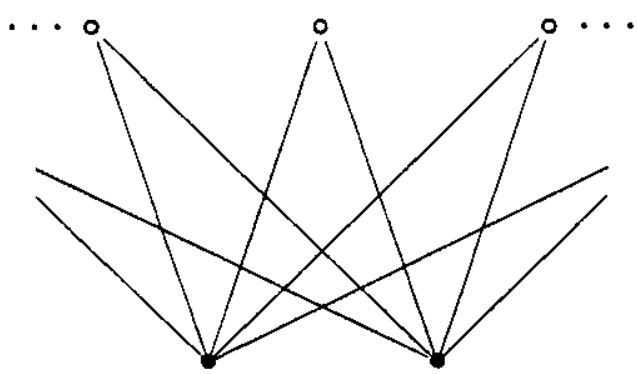

b.

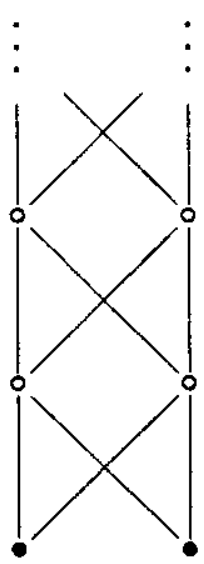

c.

Figure 1: Posets that are not Plotkin orders.

bounds for $u$ which is complete for $u$ in $B$.

2. If $A \triangleleft B \triangleleft C$ then $A \triangleleft C$.

3. If $A \subseteq B \subseteq C$ and $A \triangleleft C$ then $A \triangleleft B$.

Definition: A pre-order $A$ is a Plotkin order if for every finite $u \subseteq A$, there is a finite $B \supseteq u$ such that $B \triangleleft A$. The category of Plotkin orders with approximable relations will be denoted by PLT.

Intuitively, if $S \triangleleft A$ then $S$ offers a directed approximation to every element of $A$. Thus one might think of $S$ as itself an approximation to $A$. A pre-order $A$ is a Plotkin order just in case it can be built up as a union of finite approximations. Obviously any finite pre-order is a Plotkin order. There are a couple of similar conditions on pre-orders which are frequently useful. An upper bound $X \vdash u$ of $u$ is minimal if for each $Y, X \vdash Y \vdash u$ implies $X \sim Y$. If every finite subset of $A$ has a complete set of minimal upper bounds then we say that $A$ has the (weak) minimal upper bounds property (or "property $\mathrm{m}$ "). If every finite subset of $A$ has a finite complete set of minimal upper bounds then we say that $A$ has the strong minimal upper bounds property (or "property $\mathrm{M}^{\prime \prime}$ ). Any pre-order which has property $\mathrm{M}$ and no ascending chains is a Plotkin order. A proof of this uses König's lemma and can be found in [21]. Let $A$ be a poset and suppose $u \subseteq A$ is finite. If a complete set $u^{\prime}$ of upper bounds of $u$ is finite then it contains a complete set of minimal upper bounds. If $A$ is a Plotkin order then there is a finite $B \triangleleft A$ with $u \subseteq B$. Hence, by Lemma 6, $u$ has a finite set of minimal upper bounds in $A$. It follows, therefore, that a Plotkin order has property $M$. It is not true, however, that every pre-order having property $M$ is a Plotkin order. $A$ counter-example is illustrated in Figure 1a. Figures $1 \mathrm{~b}$ and $1 \mathrm{c}$ illustrate two other ways in which a poset can fail to be a Plotkin order (by failing to have property $M$ ).

It is often easier to work with Plotkin orders which are posets. Little is lost by this restriction, since every pre-order is isomorphic (in the category with approximable relations as arrows) to a poset $\tilde{A}$ and it is possible to show that $A$ is a Plotkin order if and only if $\tilde{A}$ is a Plotkin poset. We might have taken the Plotkin posets as our fundamental notion but this would complicate the 
definitions of some operators, and in any event would narrow the scope of discussion unnecessarily. We will, however, sometimes restrict attention to posets in order to simplify the discussion.

Suppose $A$ is a pre-order. For each $u \subseteq A$, let

$$
\operatorname{MUB}_{A}(u)=\{X \in A \mid X \text { is a minimal upper bound of } u\} \text {. }
$$

For each $S \subseteq A$, we define subsets $U_{A}^{n}(S) \subseteq A, n \in \omega$, as follows:

$$
\begin{aligned}
& U_{A}^{0}(S)=S, \\
& U_{A}^{n+1}(S)=\left\{X \mid X \in \operatorname{MUB}_{A}(u) \text { for some finite } u \subseteq U_{A}^{n}(S)\right\}, \\
& u_{A}^{*}(S)=\bigcup_{n \in \omega} u_{A}^{n}(S) .
\end{aligned}
$$

As usual, when $A$ is understood from context we drop the subscripts.

Lemma 7: If $A$ is a poset with property $\mathrm{m}$ and $S \subseteq A$, then

$$
U^{*}(S)=\bigcap\{B \mid S \subseteq B \triangleleft A\} \triangleleft A
$$

Thus, $A$ is a Plotkin poset if and only if $A$ has property $\mathrm{m}$ and for every finite $u \subseteq A, U^{*}(u)$ is finite.

Proof. Suppose $S \subseteq B \triangleleft A$. Then clearly $S=U^{0}(S) \subseteq B$. So suppose $\mathcal{U}^{n}(S) \subseteq B$ and $X \in \operatorname{MUB}(u)$ for some finite $u \subseteq U^{n}(S)$. Since $B \triangleleft A$, there is a $Y \in B$ such that $X \sqsupseteq Y$ and $Y \sqsupseteq u$. But this means $Y=X$ so $X \in B$. Hence $U^{n+1}(S) \subseteq B$ and we conclude that $U^{*}(S) \subseteq B$. To sce that $U^{*}(S) \triangleleft A$, let $u \subseteq U^{*}(S)$ be finite. Then $u \subseteq U^{n}(S)$ for some $n$. So, if $X \sqsupseteq u$ then $X \sqsupseteq Y$ for some $Y \in \operatorname{MUB}(u) \subseteq U^{n+1}(X) \subseteq u^{*}(S)$.

Definition: Let $A$ and $B$ be pre-orders. We define the exponential pre-order $\left\langle B^{A}, \vdash_{B^{A}}\right\rangle$ as follows:

1. $p \in B^{A}$ if and only if $p$ is a finite non-empty subset of $A \times B$ such that for every $Z \in A$, the set $\left\{(X, Y) \in p \mid Z \vdash_{A} X\right\}$ has a maximum with respect to the ordering on $A \times B$.

2. $p \vdash_{B^{A}} q$ if and only if for every $(X, Y) \in q$ there is a pair $\left(X^{\prime}, Y^{\prime}\right) \in p$ such that $X \vdash_{A} X^{\prime}$ and $Y^{\prime} \vdash_{B} Y$.

The intuition behind the exponential is that each $p \in B^{A}$ is a finite piece of an approximable relation. The complexity of 1 is due to the fact that $p$ must be "complete" enough to fully specify what is happening at the minimal upper bounds of finite subsets of its domain. Note that if $p \in B^{A}$ then $\{X \mid(X, Y) \in p\} \triangleleft A$. Perhaps it is more intuitive to understand the elements of $B^{A}$ in terms of the familiar concept of a step function. If $p \in B^{A}$, define $\operatorname{step}_{p}: \tilde{A} \rightarrow \tilde{B}$ by

$$
\operatorname{step}_{p}(\tilde{Z})=\max \{\tilde{Y} \mid Z \vdash X \text { and }(X, Y) \in p\} \text {. }
$$

Then $\operatorname{step}_{p}$ is a monotone function and for each $p, q \in B^{A}$, step $p_{p} \sqsupseteq \operatorname{step}_{q}$ if and only if $p \vdash_{B^{A}} q$.

If we "order" the posets with the relation $\triangleleft$ then we come quite close to getting a dcpo. The relation $\triangleleft$ is reflexive (on posets), anti-symmetric and transitive. Moreover, if $M$ is a collection 
of posets which is directed with respect to $\triangleleft$ then $\cup M$ is the least upper bound of $M$. The only reason that the posets with $\triangleleft$ fail to be a dcpo is that the posets form a proper class-not a set. When we think about $\mathbf{P O}$ as ordered by $\triangleleft$ then we lose anti-symmetry. But this is a small matter, the following definitions of monotone and continuous operators still seem quite natural.

Definition: Suppose $\mathbf{C} \subseteq$ PO. Let us say that an operator $F: \mathbf{C} \rightarrow \mathbf{C}$ is monotone if for every pair of pre-orders $A \triangleleft B$, we have $F(A) \triangleleft F(B)$. A monotone operator is continuous if for every pre-order $A$ and dirccted set $\Im$ of normal substructures of $A$ such that $A=\bigcup \Im$, we have $F(A)=$ $\bigcup\{F(B) \mid B \in \Im\}$.

It is possible to link continuity in the sense of the above definition to continuity in the categorical sense by thinking of the pre-orders as a category with the relations $\triangleleft$ as arrows. Then the monotone operators are functors and the continuous operators are functors which preserve direct limits. Later we show how to find fixed points for continuous operators in a manner analogous to that used for finding fixed points of continuous functions. But there is another use of the continuity condition on operators given by the following

Theorem 8: If $F: \mathrm{PO} \rightarrow \mathrm{PO}$ is a continuous operator and $F(A)$ is finite whenever $A$ is finite then $F$ cuts down to an operator on PLT, i.e. $F(A)$ is a Plotkin order whenever $A$ is a Plotkin order.

Proof. Suppose $A$ is a Plotkin order and $u \subseteq F(A)$ is finite. Let $\Im=\{B \triangleleft A \mid B$ is finite $\}$. Since $A$ is a Plotkin order, this set is directed and $\bigcup \Im=A$. Hence, by the continuity of $F, F(A)=\bigcup M$ where $M=\{F(B) \mid B \in \Im\}$. Since $u$ is finite and $M$ is directed, there is a $B \triangleleft A$ such that $u \subseteq F(B)$. But $F(B)$ is finite since $B$ is and $F(B) \triangleleft F(A)$ since $F$ is monotone. Hence $F(A)$ is a Plotkin order.

The definition and theorem can be extended in a straight-forward way to include multiary operators. If $F: \mathbf{P O}^{n} \rightarrow \mathbf{P O}$ then say that $F$ is monotone (continuous) if it is monotone (continuous) in each of its $n$ coordinates. If $F: \mathbf{P O}^{n} \rightarrow \mathbf{P O}^{m}$ by

$$
F\left(A_{1}, \ldots, A_{n}\right)=\left(G_{1}\left(A_{1}, \ldots, A_{n}\right), \ldots, G_{m}\left(\dot{A}_{1}, \ldots, A_{n}\right)\right)
$$

then say that it is monotone (continuous) if $G_{i}$ is monotone (continuous) for each $i=1, \ldots, m$. It is casy to check that composition of operators preserves monotonicity and continuity.

Theorem 9: The product and coproduct operators are continuous and send finite pre-orders to finite pre-orders. Hence they cut down to operators on PLT.

The proof of the Theorem is not difficult. Unfortunately, Theorem 8 is not quite general enough to apply to the exponential operator. So we treat the exponential separately below. The following lemma is technically useful and helps pin down the intuition behind the definition of $B^{A}$.

Lemma 10: If $f: A \rightarrow B$ is approximable and $M \triangleleft A, N \triangleleft B$ are finite then $f \cap(M \times N)$ is an element of $B^{A}$. 
Proof. Let $X \in A$. Since $M \triangleleft A$ there is an $X_{0} \in M$ such that $X \vdash_{A} X_{0} \vdash_{A} M \cap \downarrow X$. If $v=\left\{Y \in N \mid X_{0} f Y\right\}$ then because $f$ is approximable, there is a $Y \in B$ such that $Y \vdash_{B} v$ and $X_{0} f Y$. Since $N \triangleleft B$ there is a $Y_{0} \in N$ such that $Y \vdash_{B} Y_{0} \vdash_{B} N \cap \downarrow Y$ But $f$ is approximable so $X_{0} f Y_{0}$.

Theorem 11: Let $A$ und $B$ be pre-orders. Then

1. If $M \triangleleft A$ and $N \triangleleft B$ are finite then $N^{M} \triangleleft B^{A}$.

2. If $A$ and $B$ are Plotkin orders, then $B^{A}$ is a Plotkin order.

Proof. 1. Let $p \in B^{A}$ and set $q=\left\{(X, Y) \in M \times N \mid X f_{p} Y\right\}$ where

$$
f_{p}=\left\{\left(X^{\prime}, Y^{\prime}\right) \in A \times B \mid X^{\prime} \vdash_{A} X \text { and } Y \vdash_{B} Y^{\prime} \text { for some }(X, Y) \in p\right\} .
$$

We check the three conditions for approximablility of $f_{p}$. First, if $X \in A$ then there is an $\left(X^{\prime}, Y^{\prime}\right) \in p$ such that $X \vdash_{A} X^{\prime}$. Hence $X f_{p} Y^{\prime}$ For the second condition, suppose $X f_{p} Y_{0}$ and $X f_{p} Y_{1}$. Let $\left(X_{0}^{\prime}, Y_{0}^{\prime}\right),\left(X_{1}^{\prime}, Y_{1}^{\prime}\right) \in p$ be such that $X \vdash_{A} X_{0}^{\prime}, X_{1}^{\prime}$ and $Y_{0}^{\prime} \vdash_{B} Y_{0}$ and $Y_{1}^{\prime} \vdash_{B} Y_{1}$. Since $p \in B^{A}$ there is a pair $\left(X^{\prime}, Y^{\prime}\right) \in p$ such that $X \vdash_{A} X^{\prime}$ and $X^{\prime} \vdash_{A} X_{0}^{\prime}, X_{1}^{\prime}$ and $Y^{\prime} \vdash_{B} Y_{0}^{\prime}, Y_{1}^{\prime}$. Hence $X f_{p} Y^{\prime}$ and $Y^{\prime} \vdash_{B} Y_{0}, Y_{1}$. To get the third condition, note that if $X \vdash_{A} X^{\prime}$ and $X^{\prime} f_{p} Y^{\prime}$ and $Y^{\prime} \vdash_{B} Y$ then $X f_{p} Y$ follows immediately from the definition of $f_{p}$. Since $f_{p}$ is approximable, $q \in B^{A}$ by Lemma 10. It follows directly from the definition of $q$ that $p \vdash_{B^{A}} q$. If $p \vdash_{B^{A}} r$ and $r \in N^{M}$ then $r \subseteq q$ so $q \vdash_{B^{A}} r$. Hence $N^{M} \triangleleft B^{A}$.

2. Suppose $u$ is a finite subset of $B^{A}$. Since $A$ and $B$ are Plotkin orders, there are finite subsets $M \triangleleft A$ and $N \triangleleft B$ such that

$$
\begin{aligned}
& \{X \mid(X, Y) \in u \text { for some } Y \in B\} \subseteq M, \text { and } \\
& \{Y \mid(X, Y) \in u \text { for some } X \in A\} \subseteq N .
\end{aligned}
$$

By 1., $N^{M} \triangleleft B^{A}$. Since $u \subseteq N^{M}$ and $N^{M}$ is finite the result follows.

We now arrive at the central fact about the exponential and product on PLT.

Definition: A bicartesian category $\mathbf{C}$ is closed if there is a (specified) binary operation $B^{A}$ such that for any triple $A, B, C$ of $C$-objects there is an arrow apply: $C^{B} \times B \rightarrow C$ such that for every $f: A \times B \rightarrow C$ there is a unique arrow curry $(f): A \rightarrow C^{B}$ which makes the following diagram commute.

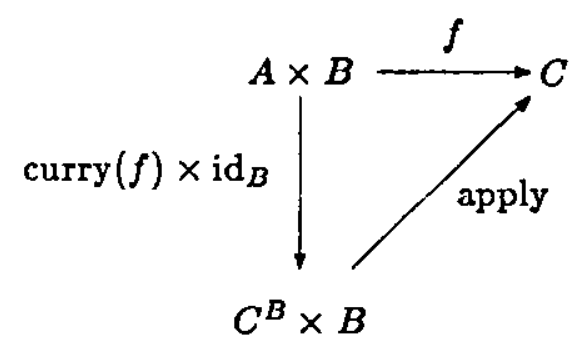


Theorem 12: The category PLT is bicartesian closed.

Proof. We have already shown that $\mathbf{P O}$ is bicartesian. By Theorem 9 the product and coproduct are endofunctors on PLT. Since 0 and 1 are finite they are Plotkin orders so PLT must be bicartesian. Theorem 11 says that the exponential is defined on PLT. To complete the proof we must demonstrate the maps curry and apply. For pre-orders $B$ and $C$, define apply $\subseteq\left(C^{B} \times B\right) \times C$ by

$$
(p, X) \text { apply } Y \text { iff } \exists\left(X^{\prime}, Y^{\prime}\right) \in p . X \vdash_{A} X^{\prime} \text { and } Y^{\prime} \vdash_{B} Y \text {. }
$$

Suppose $X \in A$ and $p \in C^{B}$. If $f: A \times B \rightarrow C$, define curry $(f)$ by

$$
X \operatorname{curry}(f) p \text { iff } \forall(Y, Z) \in p .(X, Y) f Z \text {. }
$$

One can show that curry and apply are approximable. To see that apply $\circ\left(\operatorname{curry}(f) \times \mathrm{id}_{B}\right)=f$, take $(X, Y) \in A \times B$ and $Z \in C$ such that $(X, Y) f Z$. Using the fact that $C$ and $B$ are Plotkin orders one can show that there is a $p \in C^{B}$ with $(Y, Z) \in p \subseteq f$. Thus $X$ curry $(f) p$ and $(p, Y)$ apply $Z$, so

$$
(X, Y) \text { apply } \circ\left(\operatorname{curry}(f) \times \operatorname{id}_{B}\right) Z \text {. }
$$

On the other hand, suppose equation (*) holds. Then there is a $p \in C^{B}$ such that $X$ curry $(f) p$ and $(p, Y)$ apply $Z$. By the definition of apply, there is a pair $\left(Y^{\prime}, Z^{\prime}\right) \in p$ such that $Y \vdash_{B} Y^{\prime}$, $Z^{\prime} \vdash_{C} Z$ and $\left(X, Y^{\prime}\right) f Z^{\prime}$. Now, $X$ curry $(f) p$ implies $\left(X, Y^{\prime}\right) f Z^{\prime}$. Hence $(X, Y) f Z$. We leave the proof that curry $(f)$ is unique to the reader.

Corollary 13: If $A$ and $B$ are Plotkin orders, then $\left.\left|B^{A}\right| \cong \| A|\rightarrow| B \mid\right]$.

Proof. By Theorem 4, $[|A| \rightarrow|B|] \cong \mathbf{P O}(A, B)$. It is also clear that $\mathrm{PO}(A, B) \cong \mathrm{PO}(1 \times A, B)$ and $\mathrm{PO}\left(1, B^{A}\right) \cong\left|B^{A}\right|$. By Theorem 12, curry : $\mathrm{PO}(1 \times A, B) \rightarrow \mathrm{PO}\left(1, B^{1}\right)$ is a bijection with inverse $g \mapsto$ apply $\circ(g \times$ id $)$. The fact that curry and its inverse are monotone follows immediately from their definitions so we have the desired isomorphism.

The assumption in the corollary that $A$ and $B$ are Plotkin orders is important. The result does not hold for all pre-orders. In fact, we mention later a partial converse to the corollary.

\section{PROFINITE DOMAINS.}

Categorically speaking, a dcpo is profinite if it is isomorphic to an inverse limit of finite posets in the category of dcpo's with projections as arrows. We explain shortly what a projection is but we hope to circumvent the use of this categorical definition in favor of notions which are more elementary and intrinsic. Profinite domains with a countable basis and bottom element are called strongly algebraic domains. With continuous functions as arrows they form a cartesian closed category called SFP which was introduced by Gordon Plotkin [11]. Plotkin needed a class of semantic domains closed under the powerdomain operation which he used to provide a denotational semantics for a particular multiprocessing language. To the reader familiar with strongly algebraic 
domains, a conntably bissed protinite domiain is a poset which is isomorphic to a scott compact open subset of a strongly algebraic domain. In other words, a poset $D$ is $\omega$-protinite if and only if there is a strougly algebraic poset $E$ ancl a finito set $u \subseteq \mathbf{B}[E]$ such that $E \cong\{x \in D \mid x \sqsupseteq y$ for some $y \in u\}$. Thus, if $D$ is $\omega$-profinite then the lift ${ }^{3} D_{\perp}$ of $D$ is strongly algebraic. However. it is not true, in general, that if $D_{\perp}$ is strongly algebraic then $D$ is $\omega$-profinite.

Before we give a proper definition of what a profinite domain is, we mention the notion of a projection. Let $D$ and $E$ be depo's. A projection-embedling pair is a pair $\langle p, q\rangle$ of continuous maps $p: E \rightarrow D$ and $q: D \rightarrow E$ such that $p \circ q=\mathrm{id}_{D}$ and $q \circ p \sqsubseteq \mathrm{id}_{E}$. The function $p$ is the projection and $q$ is the embedding. We abbreviate by writing $\langle p, q\rangle: E \stackrel{p e}{\longrightarrow} D$. In this section wo look at the relationship between normal substructures of pre-orders and pe-pairs from the point of view of approximable relations. We thereby generalize the theory exposited in [17] to the category of algebraic depo's. These results will be used to derive a universal domain technique for the Plotkin orders. Let $A$ and $B$ be pre-orders. Write $A \unlhd B$ if there is an $A^{\prime} \triangleleft B$ such that $A \cong A^{\prime}$.

Theorem 14: Let $A$ and $B$ be pre-orders.

1. Suppose $A \triangleleft B$ and $\vdash$ is the order relation on $B \times B$. If $p=(B \times A) \cap \vdash$ and $q=(A \times$ $B) \cap \vdash$ then $p, q$ are approximable relations, $p \circ q=\mathrm{id}_{A}$ and $q \circ p \subseteq \mathrm{id}_{B}$. In other words $\langle|p|,|q|\rangle:|B| \stackrel{\text { pe }}{\longrightarrow}|A|$.

2. Conversely, if $\langle|p|,|q|\rangle:|B| \stackrel{\text { pe }}{\longrightarrow}|A|$ for approximable relations $p$ and $q$ then $A \unlhd B$. In particular, $A \cong\{Y \in B \mid Y(q \circ p) Y\} \triangleleft B$.

Proof. The proof of 1 is a straight-forward verification. To prove 2, we begin by showing that $A^{\prime} \triangleleft B$. Suppose $u \subseteq A^{\prime}$ is finite and $Z \vdash u$. For each $X \in u$, there is an $X^{\prime} \in A$ such that $X p X^{\prime} q X$. Let $v=\left\{X^{\prime} \mid X \in u\right\}$. Then $Z p X^{\prime}$ for each $X^{\prime} \in v$ so there is a $Y \in A$ such that $Z p Y \vdash v$. Now, $Y p \circ q Y$ so there is a $Z^{\prime} \in B$ such that $Y q Z^{\prime} p Y$. But then $Z^{\prime} p Y q Z^{\prime}$ so $Z^{\prime} \in A^{\prime}$. If $X \in u$ then $Y \vdash X^{\prime}$ so $Y q X$. Since $Z^{\prime} p Y$ we get $Z^{\prime} q \circ p X$ and therefore $Z^{\prime} \vdash X$. Moreover, $Z$ p $Y q Z^{\prime}$ so $Z \vdash Z^{\prime}$.

Let $p^{\prime}=p \cap\left(A^{\prime} \times A\right)$ and $q^{\prime}=q \cap\left(A \times A^{\prime}\right)$. That $p^{\prime}$ is approximable follows immediately from the approximability of $p$. If $X \in A$ and $X q^{\prime} Y, Y^{\prime}$ for $Y, Y^{\prime} \in A^{\prime}$ then $X q Z$ for some $Z \in B$ such that $Z \vdash Y, Y^{\prime}$. Since $A^{\prime} \triangleleft B$, there is a $Z^{\prime} \in A^{\prime}$ such that $Z \vdash Z^{\prime} \vdash Y, Y^{\prime}$. Hence $X q^{\prime} Z^{\prime}$. The other conditions are easy to check. Now, suppose $X \in A$. Then $X p \circ q X$ so $X q Y p X$ for some $Y \in B$. But then $Y \in A^{\prime}$ so $X p^{\prime} \circ q^{\prime} X$. Since $p^{\prime} \circ q^{\prime} \subseteq \mathrm{id}_{A}$, we conclude that $p^{\prime} \circ q^{\prime}=\mathrm{id} A$. Suppose on the other hand, that $Y \in A^{\prime}$. Then, by definition, $Y q \circ p Y$. Since $q^{\prime} \circ p^{\prime} \subseteq \mathrm{id}_{A^{\prime}}$ we must have $q^{\prime} \circ p^{\prime}=\mathrm{id}_{A^{\prime}}$. This proves the desired isomorphism.

Theorem 15: Suppose $A$ is a pre-order and $f: A \rightarrow A$ is an approximable relation such that $f \circ f=$ $f \subseteq \mathrm{id}_{A}$. Then the following are equivalent:

\section{1. $\operatorname{im}(|f|)$ is algebraic.}

\footnotetext{
${ }^{3}$ The lift of $D$ is obtained by attaching a new element $\perp$ to $D$ which is taken to lie below each of the elements of $D$.
} 
2. For each $X, Z \in A$, if $X f Z$ then $X \vdash Y f Y \vdash Z$ for some $Y \in A$.

Proof. (1) $\Rightarrow(2)$. Suppose $X f Z$. Then $Z \in|f|(\downarrow X)$ and since $i m(|f|)$ is algebraic there is a finite $x \in \operatorname{im}(|f|)$ such that $Z \in x \subseteq|f|(\downarrow X)$. But $x$ is finite in $|A|$ so $x=\downarrow Y$ for some $Y$. This $Y$ has the property in the conclusion of (2).

$(2) \Rightarrow(1)$. Certainly, if (2) holds then $|f|$ is a deflation. To see that it has an algebraic image, note that if $X f X$ then $\downarrow X=|f|(\downarrow X)$ so $\downarrow X$ is a finite element of $i m(|f|)$. If $x \in|A|$ then

$$
\begin{aligned}
|f|(x) & =\{Z \mid X f Z \text { some } X \in x\} \\
& =\{Z \mid X \vdash Y f Y \vdash Z \text { some } X \in x \text { and some } Y\} \\
& =\bigcup\{\downarrow Y \mid Y \in x \text { and } Y f Y\} .
\end{aligned}
$$

To see that this set is directed, suppose $X f X$ and $Y$ f $Y$. If $Z f X, Y$ then $Z f Z^{\prime} \vdash X, Y$ for some $Z^{\prime}$. Hence $Z \vdash W f W \vdash Z^{\prime} \vdash X, Y$ for some $W$. We conclude that $i m(|f|)$ is algebraic.

Definition: If $A$ is a poset then we denote by $N(A)$ the set of normal substructures of $A$, ordered by set inclusion.

Proposition 16: Let $A$ be a poset. Then $N(A)$ is a dcpo. If $A$ has property $\mathrm{m}$ then $N(A)$ has a least element called the root of $A$. It is given by the equation $\operatorname{rt}(A)=\bigcap\{B \mid B \triangleleft A\}$.

Proof. Suppose $M \subseteq N(A)$ is directed and $X \in A$. If $u \subseteq \downarrow X \cap \cup M$ is finite then $u \subseteq B$ for some $B \in M$. Since $B \triangleleft A$ there is an $X^{\prime} \in B$ such that $X \vdash X^{\prime} \vdash u$. Hence $\cup M \in N(A)$. Obviously, $\cup M$ is the least upper bound of $M$. Now suppose $A$ has property m. Note that if $u \subseteq \operatorname{rt}(A)$ is finite then the complete set $u^{\prime}$ of minimal upper bounds of $u$ is in $B$ for each $B \triangleleft A$. Hence $\operatorname{rt}(A) \triangleleft A$. $\operatorname{rt}(A)$ is evidently the least member of $N(A)$.

Actually, if $A$ has property $m$ then $N(A)$ is an algebraic lattice. And if $A$ is a Plotkin poset then $N(A)$ is a locally finite algebraic lattice; that is, $\left\{x_{0} \in \mathbf{B}[N(A)] \mid x_{0} \sqsubseteq x\right\}$ is finite for each $x \in \mathbf{B}[N(A)]$.

Lemma 17: If $i: A \unlhd B$ then $N(i): N(A) \rightarrow N(B)$ given by $N(i)\left(A^{\prime}\right)=\left\{i(X) \mid X \in A^{\prime}\right\}$ is continuous.

A profinite domain may be defined in the following way.

Definition: Let $D$ be a depo and let $M$ be the set of continuous functions $p: D \rightarrow D$ such that $p=p \circ p \sqsubseteq \mathrm{id}_{D}$ and $i m(p)$ is finite. Then $D$ is profinite if $M$ is directed and $U M=\mathrm{id}_{D} . \square$

Theorem 18: $A$ dcpo $D$ is profinite if and only if $D$ is algebraic and $\mathbf{B}[D]$ is a Plotkin order. So the cateogry of profinite domains and continuous functions is equivalent to PLT. Similarly, the category of countably based profinite domains is equivalent to the category wPLT of countable Plotkin orders.

The proof uses Theorem 14. Smyth [21] shows that an $\omega$-algebraic depo $D$ with a least element is strongly algebraic if and only if $[D \rightarrow D]$ is $\omega$-algebraic. This shows that SFP is the largest cartesian closed (full) subcategory of the $\omega$-algebraic dcpo's with bottoms. A proof similar to Smyth's can be used to show the following. 


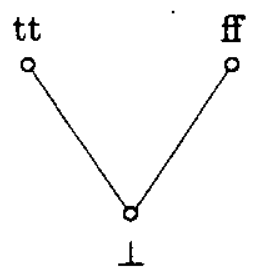

Figure 2: The truth value dcpo.

Theorem 19: If $D$ is w-algebraic and $[D \rightarrow D]$ is $\omega$-algebraic then $D$ and $[D \rightarrow D]$ are $\omega$ profinite.

A rather obvious consequence of the thcorem is that the largest cartesian closed category of (bottomless) w-algebraic dcpo's has coproducts-a property which SFP does not have. In fact there are a great many interesting bicartesian closed proper subcategories of the profinite domains. Scott has observed that a cartesian closed category $\mathbf{C}$ of dcpo's with bottom elements induces a bicartesian closed category $\mathbf{C}^{\prime}$ as follows. The arrows of $\mathbf{C}^{\prime}$ arc continuous functions and a dcpo $D$ is an object of $\mathbf{C}^{\prime}$ if $D \cong D_{1}+\cdots+D_{n}$ for some $n$ and objects $D_{1}, \ldots, D_{n}$ of $\mathbf{C}$. That $\mathbf{C}^{\prime}$ is bicartesian closed is proved by using the natural isomorphisms invloving,$+ \times, \rightarrow$ which hold for dcpo's and noting that the equation $(E+F)^{D} \cong E^{D}+F^{D}$ holds when $D$ is a depo with a least element. The profinite domains do not arise in this way though; it is not hard to see that a sum of strongly algebraic domains is just an $\omega$-profinite domain with a basis having a discrete root.

\section{UNIVERSAL DOMAINS.}

We now investigate the mathematical problem of the existence of a profinite universal domain. In the literature there are three primary examples of universal domains. The simplest is the so-called graph model $P \omega$ which is the algebraic lattice of subsets of $\omega$ ordered by set inclusion. It receives a detailed study in [15] where it is proved that any countably based algebraic lattice is a retract of $P \omega .^{4}$ Some domain theorists felt, however, that for applications in denotational semantics of programming languages it would be easier to use a class which did not require the existence of a largest (top) element. Plotkin [13] showed that the poset $T^{\omega}$ of functions from $\omega$ into the truth value dcpo $T$ (see figure 2) is universal in the sense that every coherent $\omega$-algebraic dcpo is a retract of $T^{\omega}$. Since $T^{\omega}$ is itself algebraic and coherent this provided a universal domain for a class of algebraic dcpo's that included the algebraic lattices but contained also certain desired dcpo's without tops. In [16], [18], and [17] yet a third universal domain $U$ is discussed. Although $U$ is harder to understand than $P_{\omega}$ or $T^{\omega}$ it has the advantage of having every bounded complete $\omega$-algebraic dcpo as a projection (not just as a retract). There are instances in which a "retraction

\footnotetext{
${ }^{4} A$ continuous function $r: E \rightarrow D$ is said to be a retraction if there is a continuous function $r^{\prime}: D \rightarrow E$ (called a section) such that $r \circ r^{\prime}=\mathrm{id}_{D}$. If there is a retraction $r: E \rightarrow D$ then $D$ is said to be a retract of $E$.
} 
universal" domain does not have all of the desired properties so that a "projection universal" domain is needed. For example Mulmuley [10] requires a projection universal domain to prove some of his results on the existence of inclusive predicates (for showing equivalence of semantics). Table 1 lists some of the known results on universal domains. Posets in the left column are assumed to be countable; their ideal completions are countably based.

Elementary proofs of the universality of $U$ appear in [16] and in [1]. A less elementary proof which uses results from the previous section can be carried out as follows. Let $B$ be the countable atomless boolean algebra and suppose $A$ is a countable bounded complete poset. Now, $A$ can be embedded into a countable boolean algebra in a way that preserves existing joins in $A$ and such that the join of the image of an unbounded subset of $A$ is the top element. But any countable boolean algebra is isomorphic to a subalgebra of $B$. Thus $A \unlhd B^{-}$where $B^{-}$is $B$ minus its top element. We conclude that if $A$ is countable and bounded complete then there is a continuous projection $p:\left|B^{-}\right| \rightarrow|A|$. Thus $U=\left|B^{-}\right|$is universal for the bounded complete algebraic dcpo's.

In what follows we use a technique similar to the one for $U$ to get universal domains for certain classes of $\omega$-profinite domains. If $A$ is a poset with property $m$ then we remarked in Lemma 16 that $\operatorname{rt}(A)$ is the least element in $N(A)$. Now, if $A$ and $B$ are Plotkin posets and $A \unlhd B$ then $\operatorname{rt}(A) \cong \operatorname{rt}(B)$ so by Theorem 14 no profinite domain can be a continuous projection of a profinite domain that has a different root. Hence there cannot be a projection universal $\omega$-profinite domain. We prove the next best thing: for each finite poset $A \cong \operatorname{rt}(A)$ there is a countable Plotkin poset $V_{A}$ such that if $B$ is a countable Plotkin poset with $\operatorname{rt}(B) \cong A$ then $B \unlhd V_{A}$. A fairly detailed outline of one technique of construction is offered here and we mention a second (closely related) technique. Kamimura and Tang [4] use a different approach to get a retraction universal model for the $\omega$-profinite domains having bottoms. Their model, like $P_{\omega}$ and $T^{\omega}$, is locally finite but is somewhat less natural than either of those models. In the opinion of the author, however, the construction described below does the most to revcal the fundamental idea that gives the existence result and yields the most detailed description of the model being built. (We are even able to draw a partial picture of it!) We begin by stating an interesting structure theorem for Plotkin posets.

Proposition 20: If $A$ and $B$ are finite posets such that $A \triangleleft B$ but $A \neq B$ then there are posets $A_{0}, \ldots, A_{n}$ such that

$$
A=A_{0} \triangleleft A_{1} \triangleleft \cdots \triangleleft A_{n-1} \triangleleft A_{n}=B
$$

and for each $k<n, A_{k+1}-A_{k}$ is a singleton.

Proof. If $B-A$ is a singleton then we are done. Assume that the result holds for any pair $A^{\prime} \triangleleft B^{\prime}$ such that $B^{\prime}-A^{\prime}$ has fewer that $n$ elements. Suppose there are $n$ elements in $B-A$ and let $X$ be a maximal clement of $B-A$, i.e. if $Y \in B$ such that $X \sqsubset Y$ then $Y \in A$. Set $A^{\prime}=A \cup\{X\}$. We show that $A^{\prime} \triangleleft B$. Let $Z \in B$ and suppose

$$
u=\left\{Y \in A^{\prime} \mid Y \sqsubseteq Z\right\}
$$

We must demonstrate that $u$ has a largest element. If $u \subseteq A$ then this follows from the fact that 


\begin{tabular}{c|c|c} 
POSETS & $\begin{array}{c}\text { IDEAL } \\
\text { COMPLETIONS }\end{array}$ & $\begin{array}{c}\text { UNIVERSAL } \\
\text { DOMAIN }\end{array}$ \\
\hline $\begin{array}{c}\text { Upper Semi- } \\
\text { lattices }\end{array}$ & $\begin{array}{c}\text { Algebraic } \\
\text { Lattices }\end{array}$ & $\rho_{\omega}$ \\
\hline Coherent Pre-orders & $\begin{array}{c}\text { Coherent } \\
\text { Algebraic Dcpo's }\end{array}$ & $T^{\omega}$ \\
\hline $\begin{array}{c}\text { Bounded Complete } \\
\text { Pre-orders }\end{array}$ & Bounded Complete \\
Algebraic Dcpo's & $U$ \\
\hline Plotkin Orders & Profinite Dcpo's & $?$
\end{tabular}

Table 1: Universal domains.

$A \triangleleft B$. if $X \in u$ then $X \sqsubseteq Z$ so $X=Z$ or $Z \in A$. In either case, $Z$ is the largest elernent of $u$. Hence $A^{\prime} \triangleleft B$. Since $A \triangleleft B$ we have $A \triangleleft A^{\prime} \triangleleft B$. But $B-A^{\prime}$ has $n-1$ elements, so by the induction hypothesis, there are posets $A_{1}, \ldots, A_{n}$ such that $A \triangleleft A^{\prime}=A_{1} \triangleleft \cdots \triangleleft A_{n}=B$.

Theorem 21: (Enumeration) If $A$ is a countable Plotkin poset and $B=\operatorname{rt}(A)$, then there is an enumeration $X_{0}, X_{1}, \ldots$ of $A$ such that for each $n, B \cup\left\{X_{i} \mid i<n\right\} \triangleleft A$.

Proof. Suppose $\operatorname{rt}(A)=A_{0} \triangleleft A_{1} \triangleleft \cdots$ is a chain of finite normal substructures of $A$ such that $A=\bigcup_{n \in \omega} A_{n}$. Let $B_{0} \triangleleft B_{1} \triangleleft \cdots$ be a new chain that results from deleting $A_{n+1}$ for each $n$ if it equals $A_{n}$. Using Lemma 20 we may refine this chain to a chain $C_{0} \triangleleft C_{1} \triangleleft \cdots$ such that $C_{0}=\operatorname{rt}(A)$ and for each $n, C_{n+1}-C_{n}$ is a singleton $Z_{n}$. Now, let $X_{0}, \ldots, X_{k-1}$ be an enumeration of $C_{0}$ and for each $n$, let $X_{n+k}=Z_{n}$. This enumeration has the desired property.

Definition: Let $\langle A, \sqsubseteq\rangle$ be a poset. For each $X \in A$, let $\mathbf{X}$ be a constant symbol naming $X$. Let $\preceq$ be a binary relation symbol which is interpreted by $\sqsubseteq$. A diagram type over $A$ is a set $\Gamma$ of inequalities and negations of inequalities between constant symbols and a variable $\mathrm{v}$, i.e. formulas of the form

$$
\mathbf{v} \preceq \mathbf{X}, \quad \mathbf{v} \preceq \mathbf{x}, \quad \mathbf{X} \preceq \mathbf{v}, \quad \mathbf{X} \preceq \mathbf{v}
$$

where $X \in A$. If $A \subseteq B$ and $Z \in B$ then the diagram type of $Z$ over $A$ is the set of all such equations (using constant symbols for elements of $A$ ) that hold when $v$ is given the value $Z$ and $\preceq$ is interpreted as the order relation on $B$. A diagram type $\Gamma$ over $A$ is said to be realized in $B$ by $Z$ if $\Gamma$ is a subset of the diagram type of $Z$ over $A$. A diagram type $\Gamma$ over a poset $A$ is said to be normal if there is a poset $B$ with $A \triangleleft B$ such that $\Gamma$ is realized in $B$.

Lemma 22: If $\Gamma$ is a normal type over a finite poset $B$ and $B \triangleleft A$ then there is a finite poset $A_{1}$ such that $A \triangleleft A_{1}$ and $\Gamma$ is realized by some $Z \in A_{1}$ such that $B \cup\{Z\} \triangleleft A_{1}$. 
Proof. Let $\sqsubseteq$ be the partial ordering on $A$. Since $B \triangleleft A, B$ inherits this ordering. Suppose $B \triangleleft A_{0}$ and $Z \in A_{0}$ such that $Z$ rcalizes $\Gamma$. Let $\Xi_{0}$ be the partial ordering on $A_{0}$. Note that the restriction of $\sqsubseteq_{0}$ to $B$ is the same as the restriction of $\sqsubseteq$ to $B$. Let $A_{1}=A \cup\{Z\}$ and define a binary relation $\sqsubseteq_{1}$ on $A_{1}$ as follows:

- $Z \sqsubseteq_{1} Z$,

- if $X, Y \in A$ then $X \sqsubseteq_{1} Y$ iff $X \sqsubseteq Y$,

- if $X \in A$ then $X \sqsubseteq_{1} Z$ iff there is an $X^{\prime} \in B$ such that $X \sqsubseteq X^{\prime} \sqsubseteq_{0} Z$,

- if $X \in A$ then $Z \sqsubseteq_{1} X$ iff there is an $X^{\prime} \in B$ such that $Z \sqsubseteq_{0} X^{\prime} \sqsubseteq X$.

To see that $\left\langle A_{1}, \sqsubseteq_{1}\right\rangle$ is a poset, note that $\Xi_{1}$ is just the transitive closure of $\left(\sqsubseteq \cup \sqsubseteq_{0}\right) \cap\left(A_{1} \times A_{1}\right)$. That $\sqsubseteq_{1}$ is reflexive is immediate from its definition. To see that it is anti-symmetric, suppose $X \sqsubseteq_{1} Z \sqsubseteq_{1} X$ for some $X \in A$. Then there are $X_{0}, X_{1} \in B$ such that $X \sqsubseteq X_{0} \sqsubseteq_{0} Z$ and $Z \sqsubseteq_{0} X_{1} \sqsubseteq X$. But then $X \sqsubseteq X_{0} \sqsubseteq X_{1} \sqsubseteq X$ so $X_{0}=X_{1}=X$ and therefore $X \in B$. Hence $X \sqsubseteq_{0} Z \sqsubseteq_{0} X$ implies $X=Z$ by the anti-symmetry of $\sqsubseteq_{0}$. Of course, if $X, Y \in A$ and $X \sqsubseteq_{1} Y \sqsubseteq_{1} X$ then $X=Y$ since $X \sqsubseteq Y \sqsubseteq X$.

Now, the fact that $A$ is a subposet of $A_{1}$ is built into the definition of $\complement_{1}$. To see that $A \triangleleft A_{1}$, suppose $u \subseteq A$ is finite and $u \subseteq_{1} Z$. By the definition of $\complement_{1}$, for each $X \in u$ there is an $X^{\prime} \in B$ such that $X \sqsubseteq X^{\prime} \sqsubseteq_{1} Z$. So let $u^{\prime}=\left\{X^{\prime} \mid X \in u\right\}$. Then $u^{\prime} \sqsubseteq Z$. Since $B \triangleleft A_{0}$, there is a $Z^{\prime} \in B$ such that $u^{\prime} \sqsubseteq_{0} Z^{\prime} \sqsubseteq_{0} Z$. But this implies that $u \sqsubseteq_{1} Z^{\prime} \sqsubseteq_{1} Z$ so we may infer that $A \triangleleft A_{1}$. We must show that $B \cup\{Z\} \triangleleft A_{1}$. Suppose $u \subseteq B \cup\{Z\}$ is finite and $u \sqsubseteq_{1} X$ for some $X \in A_{1}$. We must find a $Y \in B \cup\{Z\}$ such that $u \sqsubseteq_{1} Y \sqsubseteq_{1} X$. If $X=Z$ then the result is immediate-just let $Y=X$. So suppose $X \in A$. If $Z \notin u$ then we can get the desired $Y$ by using the fact that $B \triangleleft A$. If $Z \in u$ theo there is an $X^{\prime} \in B$ such that $Z \subseteq_{0} X^{\prime} \sqsubseteq X$. Thus

$$
v=(u-\{Z\}) \cup\left\{X^{\prime}\right\} \sqsubseteq X
$$

Since $B \triangleleft A$ and $v \subseteq B$, there is some $Y \in B$ such that $v \sqsubseteq Y \sqsubseteq X$. Since $Z \sqsubseteq_{0} X^{\prime} \sqsubseteq Y$ we may conclude that $Z \sqsubseteq_{1} Y$. Thus $u \sqsubseteq_{1} Y$ and we are done.

Finally, suppose $\mathrm{v} \preceq \mathrm{X}$ is in $\Gamma$ for some $X \in B$. Then $Z \sum_{0} X$ since $Z$ realizes $\Gamma$ in $A_{0}$. Hence, by definition, $Z \sqsubseteq_{1} X$. Suppose $\mathrm{v} \npreceq \mathrm{X}$ is in $\Gamma$ but $Z \sqsubseteq_{1} X$. Then $Z \sqsubseteq_{0} X$. But this contradicts the assumption that $Z$ realizes $\Gamma$ in $A_{0}$. So apparently $Z Z_{1} X$. Similarly, the other formulas in $\Gamma$ must be realized by $Z$ in $A_{1}$. $\square$

Lernma 23: Let $A$ be a finite poset. Then there is a finite poset $A^{+}$such that $A \triangleleft A^{+}$and for every subspace $B \triangleleft A$ and normal type $\Gamma$ over $B$, there is a $Z \in A^{+}$such that $Z$ realizes $\Gamma$ and $B \cup\{Z\} \triangleleft A^{+}$.

Proof. Let $\Gamma_{1}, \ldots, \Gamma_{n}$ be all of the normal types over normal subspaces of $A$. Set $A=A_{0}$ and suppose $A \triangleleft A_{k}$. Suppose $\Gamma_{k+1}$ is normal over $B \triangleleft A$. Then $B \triangleleft A_{k}$ so by Lemma 22 there is a finite poset $A_{k+1}$ such that $A_{k} \triangleleft A_{k+1}$ and $B \cup\{Z\} \triangleleft A_{k+1}$ for some $Z$ that realizes $\Gamma_{k+1}$. Set $A^{+}=A_{n+1}$. If $Z$ realizes $\Gamma_{k+1}$ in $A_{k+1}$ then it realizes it also in $A^{+}$. Moreover, $B \cup\{Z\} \triangleleft A_{k+1} \triangleleft A^{+}$. 
Theorem 24: Let $V$ be a countable Plotkin poset. Suppose that for every finite $A \triangleleft V$ and normal type $\Gamma$ over $A$, there is a realization $Z$ for $\Gamma$ such that $A \cup\{Z\} \triangleleft V$. If $B$ is a countable Plotkin order such that $\mathrm{rt}(B) \cong \operatorname{rt}(V)$ then $B ! V$.

Proof. Suppose $B$ is a countable Plotkin order such that $\operatorname{rt}(B) \cong \operatorname{rt}(V)$. We may assume that $B$ is a poset. By the Enumeration Theorem, there is an enumeration $X_{1}, X_{1}, \ldots$ of $B$ such that for each $n \in \omega$,

$$
B_{n}=\operatorname{rt}(B) \cup\left\{X_{i} \mid i<n\right\} \triangleleft B
$$

Since $B_{0}=\operatorname{rt}(B)$, there is an isomorphism $f_{0}: B_{0} \cong V_{0}$ where $V_{0}=\operatorname{rt}(V)$. We construct an $\omega$-sequence of isomorphisms $f_{n}: A_{n} \cong V_{n}$ where $V_{n} \triangleleft V, f_{n} \subseteq f_{n+1}$ and $V_{n} \subseteq V_{n+1}$.

Suppose that $f_{n}$ and $V_{n}$ are given. Now, $B_{n} \triangleleft B_{n: 1}$ so the diagram type $\Gamma$ of $X_{n}$ over $B_{n}$ must be normal. Let $\Sigma$ be the corresponding type over $V_{n}$, i.e. $\Sigma$ is obtained from $\Gamma$ by replacing any occurrence of a constant symbol for an $X \in A_{n}$ by a constant symbol for $f_{n}(X)$. Then $\Sigma$ is a normal type over $V_{n}$ so by the hypothesis on $V$, there is a realization $Y_{n} \in V$ of $\Sigma$ such that

$$
V_{n+i}=V_{n} \cup\left\{Y_{n}\right\} \triangleleft V
$$

If we define $f_{n+1}: A_{n+1} \rightarrow V_{n+1}$ by

$$
f_{n+1}(X)= \begin{cases}f_{n}(x) & \text { if } X \in A_{n} \\ Y_{n} & \text { if } X=X_{n}\end{cases}
$$

then $f_{n} \subseteq f_{n+1}$ and $f_{n+1}$ is an isomorphism. If $f=\bigcup_{n \in \omega} f_{n}$ and $V^{\prime}=\bigcup_{n \in \omega} V_{n}$ then $f: B \cong V^{\prime}$. Moreover, since $V_{n} \triangleleft V$ for each $n, V^{\prime} \triangleleft V$. Hence $B \unlhd V$.

Corollary 25: Let $A$ be a finite poset such that $A \cong \mathrm{rt}(A)$. There is a Plotkin poset $A^{\star}$ such that whenever $B$ is a countable Plotkin order with $\operatorname{rt}(B) \cong A$, then $B \unlhd A^{\star}$.

Proof. Let $A=A_{0}$ and for each $n$, define $A_{n+1}=A_{n}^{+}$. Let $A^{\star}=\bigcup_{n \in \omega} A_{n}$. Suppose $C \triangleleft A^{\star}$ is finite. Then $C \triangleleft A_{n}$ for some $n$. If $\Gamma$ is a normal type over $C$ then $\Gamma$ is realized by a $Z \in A_{n}^{+}=A_{n+1}$ such that $C \cup\{Z\} \triangleleft A_{n+1}$. Since $A_{n+1} \triangleleft A^{\star}$, the hypotheses of Theorem 24 are satisfied and the desired conclusion therefore follows.

It is possible to get the $A^{+}$in Lemma 23 by explicit construction. One way to do this is to pre-order the set $A_{t p}=\{\Gamma \mid \Gamma$ is normal over some finite $B \triangleleft A\}$ by letting $\Gamma \vdash \Sigma$ just in case there are $X, Y \in A$ such that $\mathbf{v} \preceq \mathbf{X}$ is in $\Gamma, \mathbf{Y} \preceq \mathbf{v}$ is in $\Sigma$, and $X \sqsubseteq Y$. If we let $A^{+}=\tilde{A}_{t p}$ then there is a normal substructure $A^{\prime} \triangleleft A^{+}$with $A \cong A^{\prime}$ such that for every normal type $\Gamma$ over a substructure $B \triangleleft A^{\prime}$, there is a $Z \in A^{+}$such that $B \cup\{Z\} \triangleleft A^{+}$and $Z$ realizes $\Gamma$. To get a universal domain one solves the domain equation $A=A^{+}$. Although it is somewhat tedious to check all of the details of the construction, this more order-theoretic way of doing things helps in picturing the universal domain as the limit of the posets $A \unlhd A^{+} \unlhd A^{++} \unlhd \ldots$. Figure 3 illustrates the first three stages in the construction of the universal domain with a trivial root. 


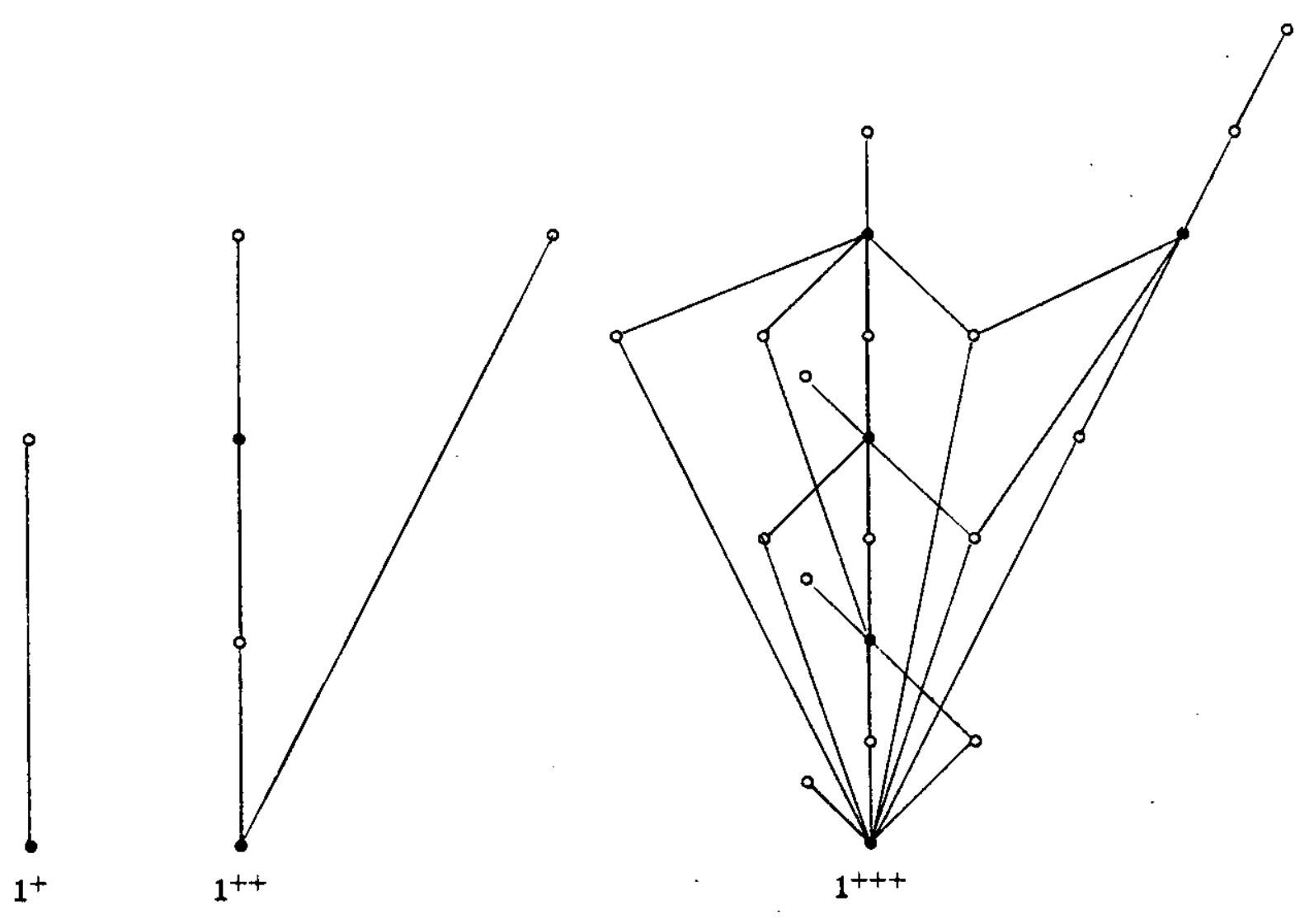

Figure 3: Construction of $1^{\star}$. 


\section{JOIN COMPLETION.}

In this section we present the join completion operator $J$. For a pre-order $A, J(A)=\{u \subseteq A \mid$ $u$ is finite and bounded $\}$ and if $u, v \in J(A)$ then

$$
u \vdash_{J(A)} v \text { iff } \forall X \in A . X \vdash_{A} u \Rightarrow X \vdash_{A} v .
$$

The following proposition lists some of the properties of $J$.

Theorem 26: Let $A$ and $B$ be pre-orders. Then

1. $\left\langle J(A), \vdash_{J(A)}\right\rangle$ is bounded complete;

2. if $A$ is bounded complete then $J(A) \cong A$;

3. $J$ is continuous;

4. $J(A \times B) \cong J(A) \times J(B)$.

Proof. (1) Suppose $u, v \in J(A)$ and $w \vdash J(A) u, v$. Then $u \cup v$ is bounded in $A$ by anything that bounds $w$. Hence $u \cup v$ is in $J(A)$ and $w \vdash_{J(A)} u \cup v$. But any bound for $u \cup v$ in $A$ is a bound for $u$ and a bound for $v$, so $u \cup v \vdash_{J(A)} u, v$. Thus $J(A)$ has bounded joins.

(2) Suppose $A$ is bounded complete and define $f \subseteq A \times J(A)$ by $X f u$ if and only if $X \vdash_{A} u$. To see that $f$ is approximable, just note that $X f u$ if and only if $X \vdash_{A} Y$ where $Y$ is a least upper bound for $u$. Hence, if $X f u, v$ then $X \vdash_{A} Y$ where $Y$ is the least upper bound of $u \cup v$ so $X f u \cup v \vdash_{J(A)} u, v$. The other conditions for approximability of $f$ are obviously satisfied. Define $g \subseteq J(A) \times A$ by $u g X$ if and only if $u \vdash_{J(A)}\{X\}$ If $u g X$ and $u g Y$ then $u g Z$ where $Z$ is a least upper bound for $u$. The remaining condition for approximability of $g$ is obviously satisfied. Now suppose $X f u$ and $u g Z$ for some $X, Z \in A$ and $u \in J(A)$. If $Y$ is a least upper bound for $u$ then $X \vdash_{A} Y \vdash_{A} Z$ so $X \vdash_{A} Z$. Therefore $g \circ f \subseteq \operatorname{id}_{A}$. If, on the other hand, $X \vdash_{A} Z$ then $X f\{X\} g Z$ so $g \circ f \supseteq \mathrm{id}_{A}$. Hence $g \circ f=\mathrm{id}_{A}$. Now, suppose $u g X$ and $X w$ for some $u, w \in J(A)$ and $X \in A$. Then $u \vdash_{J(A)}\{X\}$ and $X \vdash_{A} Y$ where $Y$ is a least upper bound of $w$. Hence $\{X\} \vdash_{J(A)}\{Y\} \vdash_{J(A)} w$ so $u \vdash_{J(A)} w$. Therefore $f \circ g \subseteq \mathrm{id}_{J(A)}$. If, on the other hand, $u \vdash_{J(A)} w$ then $u \vdash_{J(A)}\{Y\}$ for a least upper bound $Y$ of $w$ so $u g Y$ and $Y f w$ so $f \circ g \supseteq \operatorname{id} J(A)$. Hence $f \circ g=\operatorname{id} J(A)$.

(3) We must first show that if $A \triangleleft B$ then $J(A) \triangleleft J(B)$. Suppose $A \triangleleft B$. If $u$ is bounded in $A$ then it is bounded in $B$ so any element of $J(A)$ is also an element of $J(B)$. Suppose $u, v \in J(A)$ and $u \vdash_{J(A)} v$. We claim that $u \vdash_{J(B)} v$. Suppose $X \in B$ and $X \vdash_{B} u$. Since $A \triangleleft B$, there is an $X^{\prime} \in A$ such that $X \vdash_{B} X^{\prime} \vdash_{A} u$. But $u \vdash_{J(A)} v$ means $X^{\prime} \vdash_{A} v$. Hence $X \vdash_{B} v$ and the claim is established. Obviously, $u \vdash_{J(B)} v$ implies $u \vdash_{J(A)} v$. Thus $\left\langle J(A), \vdash_{J(A)}\right\rangle \subseteq\left\langle J(B), \vdash_{J(B)}\right\rangle$. To see that $J(A) \triangleleft J(B)$, suppose $u, v \in J(A)$ and $w \vdash_{J(B)} u, v$ for some $w \in J(B)$. If $X \vdash_{A} w$ for some $X \in B$ then $X \vdash_{A} u \cup v$ so $u \cup v$ is bounded and there is an $X^{\prime} \in A$ such that $X^{\prime} \vdash_{A} u \cup v$. Hence $u \cup v \in J(A)$ and we conclude that $J(A)$ is closed under existing joins in $J(B)$. Thus $J(A) \triangleleft J(B)$. To see that $J$ is continuous, suppose $B=\bigcup \Im$ where $\Im$ is a directed collection of normal substructures 
of $B$. If $u \in J(B)$ then $\bigcup u \subseteq A$ for some $A \in I$ so $u \in J(A)$. Hence $J(B) \subseteq \bigcup_{A \in \Im} J(A)$. The opposite inclusion is obvious.

(4) Left for the reader.

By Corollary 25, there is a Plotkin order $V=1^{\star}$ such that whenever $A$ is a Plotkin order with a least element, we have $A \unlhd V$. We may extract from Theorem 26 the following

Corollary 27: If $A$ is a bounded complete pre-order then $A \unlhd J(V)$.

Proof. Since $A$ has a least element we know that $A \cong A^{\prime}$ for some $A^{r} \triangleleft J(V)$. But $A^{\prime}$ is bounded complete so $A^{\prime} \cong J\left(A^{\prime}\right)$. Hence $A \cong A \unlhd J(V)$.

Now, suppose $u$ and $v$ are finite bounded subsets of $V$ such that $u, v \neq\{\perp\}$. Consider the diagram type

$$
\Gamma(\mathbf{v})=\{\perp \neq \mathbf{v}\} \cup\{\mathbf{v} \subseteq \mathbf{X} \mid X \in u \cup v\} .
$$

This type is normal over $U_{V}^{*}(u \cup v)$ so it has a realization $Z$ in $V$. But $u \vdash_{J(V)}\{Z\}, v \vdash J(V)\{Z\}$ and $\{Z\} \not\{\perp\}$. This shows that no pair $u, v \neq\{\perp\}$ of bounded subsets of $J(V)$ can be complementary to one another. Hence $J(V)$ cannot be isomorphic to the countable atomless boolcan algebra with its top element removed. We conclude that although $|J(V)|$ is projection universal for bounded complete algebraic dcpo's, it is not isomorphic to Scott's universal domain $U$.

A variant on the join completion operator has been studied independently in [3] for a different purpose. The Frink completion $\|A\|$ of a pre-order $A$ is defined there. This operation is related to the join completion by the isomorphism $\|A\| \cong\left|J(A)^{\top}\right|$ were $(\cdot)^{\top}$ is the operation that adds a new greatest element $T$.

\section{FIXED POINTS OF CONTINUOUS OPERATORS.}

In this last section we prove a theorem which gives the conditions under which a domain equation involving continuous operators has a profinite solution. Solutions to such equations over the profinite domains are more problematic than is the case for strongly algebriac domains or bounded complete algebraic domains. In these latter categories, every such equation has a solution. This is not true for the profinites because there is no initial object for the category of profinite domains and embeddings. That is, there is no profinite $D$ such that for every profinite $E$, there is a $\langle p, q\rangle: E \stackrel{\text { pe }}{\longrightarrow} D$. The single element poset 1 will not suffice, because it cannot be embedded in $1+1$ for example. The following theorem provides a reasonably simple existence condition.

Theorem 28: Suppose $F: \omega \mathrm{PLT} \rightarrow \omega \mathrm{PLT}$ is continuous. Then $F$ has a fixed point in $\omega \mathrm{PLT}$ with root isomorphic to a poset $A$ if and only if $A \cong \operatorname{rt}(F(A))$.

Proof. To prove necessity $(\Rightarrow)$, suppose $F(B) \cong B$ for a Plotkin order $B$. Then $\operatorname{rt}(B) \cong \operatorname{rt}(F(B))$. But $\operatorname{rt}(B) \triangleleft B$ so $F(\operatorname{rt}(B)) \triangleleft F(B)$ by monotonicity of $F$. Hence $\operatorname{rt}(F(\operatorname{rt}(B)))=\operatorname{rt}(F(B))$ and therefore $\operatorname{rt}(B) \cong \operatorname{rt}(F(\operatorname{rt}(B)))$. If $A \cong \operatorname{rt}(B)$ then $A \cong \operatorname{rt}(F(A))$. To prove sufficiency $(\Leftarrow)$, suppose $A \cong \operatorname{rt}(F(A))$. Then by Theorem 25 there is a pre-order $A^{*}$ and a map $i: F(A) \unlhd A^{\star}$. 
Consider the function $N(i) \circ F: N\left(A^{\star}\right) \rightarrow N\left(A^{\star}\right)$. By Lemma 17 this function is continuous so by the Tarski Fixed Point Theorem it has a least fixed point $B$. So $B=N(i)(F(B)) \cong F(B)$.

Let $P_{0}, P_{1}, \ldots$ be any enumerated collection of profinite domains such that $\operatorname{rt}\left(P_{n}\right)$ can be uniformly effectively computed from $n$. Consider the set $\mathfrak{R}$ of expressions built up from the constants $P_{n}$ and a variable $X$ using the operators $+, \times, \rightarrow,(\cdot)_{\perp}$. For example, the following expressions are in $\Re$ :

$$
[X \rightarrow X], \quad\left[X \rightarrow P_{n}\right], \quad P_{n}+[X \rightarrow X], \quad X+\left(P_{n} \times P_{m}\right)
$$

Let the expressions in $\Re$ be Gödel numbered in some effective manner. We claim that the (Gödel numbers of) expressions $E(X)$ in $\Re$ such that the equation $X \cong E(X)$ has a profinite solution is recursively enumerable. To see this, let an expression $E(X) \in \Re$ be given. Then $\operatorname{rt}(E(P))$ may be effectively computed for any finite poset $P$. If there is a finite $P$ such that $P \cong \operatorname{rt}(E(P))$ then, by Theorem 28 there is a profinite solution for $X \cong E(X)$. Since $P \cong \operatorname{rt}(E(P))$ is effectively decidable for finite posets $P$, the claim follows. We now discuss the application of the theorem to some specific equations in $\Re$.

It is possible to show that for any pair of pre-orders $A$ and $B$ having property $\mathrm{m}, \operatorname{rt}(A \times B)=$ $\operatorname{rt}(A) \times \operatorname{rt}(B)$. In light of Theorem 28 this is noteworty in the following regard. Since the product is continuous, the operator $F(A)=A \times A$ is continuous. Since $F(A)$ is finite whenever $A$ is, $F$ cuts down to a continuous operator on wPLT. Suppose $A \cong F(A)$ is a Plotkin order and let $B=\operatorname{rt}(A)$. Now, $B$ is finite so suppose it has $m$ elements. Then $\operatorname{rt}(F(A))=\operatorname{rt}(A \times A)=B \times B$ has $m^{2}$ clements. Since $\operatorname{rt}(A) \cong \operatorname{rt}(F(A))$ we must have $m=m^{2}$ so apparently $m=1$ or $m=0$. In other words, a non-empty fixed point in $\omega \mathbf{P L T}$ of the equation $A \cong F(A)$ must have a least clement. This result carries over to the $\omega$-profinite domains, because an $\omega$-profinite solution of the equation $D \cong D \times D$ gives rise to the solution $\mathbf{B}[D] \cong \mathbf{B}[D] \times \mathbf{B}[D]$ in $\omega \mathbf{P L T}$. A similar situation occurs with the diagonal of the coproduct. One can show that if $A$ and $B$ have property $\mathrm{m}$ then $\operatorname{rt}(A+B)=\operatorname{rt}(A)+\operatorname{rt}(B)$. Hence the only $\omega$-profinite solution to the equation $D \cong D+D$ is the initial object 0 .

The diagonal of the function space operator, $F(A)=A^{A}$ is more problematic, however, because it is not true in general that $\operatorname{rt}\left(B^{A}\right) \cong \operatorname{rt}(B)^{\mathrm{rt}(A)}$. Consider, for example, the opposite $T^{o p}$ of the truth value dcpo. The monotone functions from $T^{o p}$ into $T^{o p}$ form a poset whose root is not isomorphic to the poset $\left[\mathrm{rt}\left(T^{o p}\right) \rightarrow \operatorname{rt}\left(T^{o p}\right)\right]=\left[T^{o p} \rightarrow T^{o p}\right]$. Hasse diagrams for $T^{o p}$ and $\left[T^{o p} \rightarrow T^{o p}\right]$ appear in Figure 4. The root of $\left[T^{o p} \rightarrow T^{o p}\right]$ is drawn in black there.

Suppose $A$ is a non-empty finite poset and $A \cong \operatorname{rt}\left(A^{A}\right)$. We claim that $A$ is isomorphic to the trivial one element poset. To see this, suppose $A$ is not isomorphic to 0 or 1 . We may assume that $A$ is a poset; since $A$ is finite, $A^{A}$ is isomorphic to the poset $[A \rightarrow A]$ of monotone functions from $A$ into $A$. Now, $A$ has a set of $n$ minimal elements where $n>1$. A constant function mapping all of $A$ to a minimal element of $A$ is minimal in $[A \rightarrow A]$ so $\operatorname{rt}([A \rightarrow A])$ has a least $n$ minimal elements. Let $f: A \rightarrow A$ be monotone and suppose $f$ is below the identity function on $A$. Suppose $X \in A$ and $f(Y)=Y$ for every $Y \sqsubset X$. Using the fact that $A$ has no proper normal 


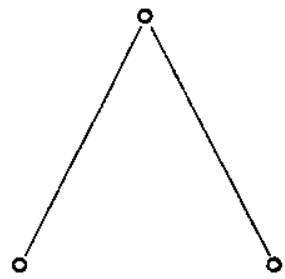

$T^{\text {op }}$

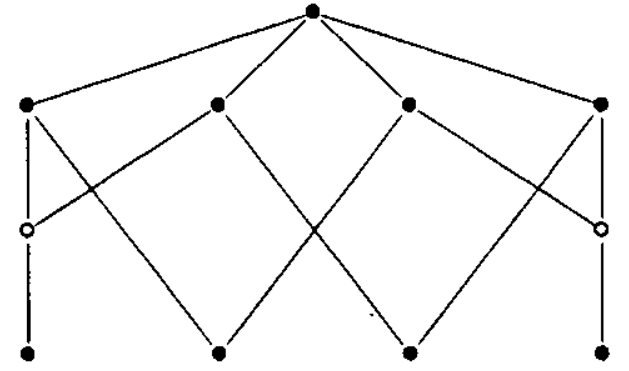

$\left[T^{o p} \rightarrow T^{o p}\right]$

Figure 4: Root of a function space.

substructure, one can show that there is a set $u \subseteq A$ such that $X$ is a minimal upper bound of $u$. But then $u=f(u) \sqsubseteq f(X) \sqsubseteq X$ so $f(X)=X$. Hence $f$ is the identity function and consequently the identity function is minimal in $[A \rightarrow A]$. Since none of the constant functions is equal to the identity function, this means $\operatorname{rt}([A \rightarrow A])$ has at least $n+1$ minimal elements. Hence, we cannot have $A \cong \operatorname{rt}([A \rightarrow A])$. This shows that a non-empty fixed point of the operator $F$ in $\omega$ PLT must have a least element. Again, this can be used to show that if $D \cong[D \rightarrow D]$ is $\omega$-profinite and non-empty then $D$ has a least element. Using Theorem 19 we get the following.

Proposition 29: If $D \cong[D \rightarrow D]$ is w-algebraic (where the possibility that $D$ has no bottom element is allowed) then it is strongly algebraic.

We conclude with a short note on powerdomains. The convex powerdomain was introduced by Plotkin [11]. Smyth [20] introduced the upper powerdomain and gave a detailed description of Plotkin's powerdomain and his using the finite elements of the domains. The definition below, which appears in Scott [19] describes these operators and the lower powerdomain through their action on pre-orders. The names for the operators are derived from mathematical considerations [21].

Definition: Let $A$ be a pre-order and suppose $M$ is the set of finite subsets of $A$. The upper powerdomain $\mathcal{Q}(A)$ of $A$ is the set $M$ together with a pre-ordering $\vdash_{\mathcal{Q}(A)}$ given by

$$
u \vdash_{\mathcal{Q}(A)} v \text { if }(\forall X \in u)(\exists Y \in v) . X \vdash_{A} Y
$$

Dually, the lower powerdomain $R(A)$ of $A$ is $M$ with the pre-ordering $\vdash_{R(A)}$ given by

$$
u \vdash_{R(A)} v \text { if }(\forall Y \in v)(\exists X \in u) . X \vdash_{A} Y .
$$

The convex powerdomain $S(A)$ of $A$ is the intersection of the upper and lower powerdomain preorderings on $M$, i.e.

$$
u \vdash_{S(A)} v \text { if } u \vdash_{\mathcal{Q}(A)} v \text { and } u \vdash_{R(A)} v
$$


If $f: A \rightarrow B$ is approximable then the action of $\mathcal{Q}, R, S$ on $f$ is given by

$$
\begin{aligned}
& u \mathcal{Q}(f) v \text { if }(\forall X \in u)(\exists Y \in v) . X f Y \\
& u R(A) v \text { if }(\forall Y \in v)(\exists X \in u) . X f Y \\
& u S(A) v \text { if } u \mathcal{Q}(f) v \text { and } u R(f) v .
\end{aligned}
$$

It is straight-forward to show that the operators $A \mapsto Q(A)$ etc. are all continuous. Since each of them obviously sends finite posets to finite posets, Theorem 8 shows that they are closed on PLT. It is well-known that the convex powerdomain does not preserve the existence of least upper bounds (-look in [11] for a counterexample). It is not closed over any of the first three classes listed in Table 1. In fact, it is rather difficult to find a cartesian closed subcategory of PO which is closed under S. PLTand some slight variants (such as the Plotkin orders having bottoms) are the only known examples.

The precise relationship between the bounded complete algebraic dcpo's and the profinites is not well understood. Although the join completion operator does provide some connection, it does not seem to be useful in resolving some of the open questions. For example, it is not known (at least to the author) whether $A \unlhd S\left(B^{-}\right)$for every countable Plotkin order $A$ with a $\perp$. By Theorem 14, this is just asking whether $|S|(U)$ is projection universal for the strongly algebraic domains (where $|S|(D)=|S(\mathbf{B}[D])|$ for algebraic dcpo's $D)$. As an aside: it is possible to show that $A \triangleleft S(A)$ for every bounded complete $A$, but the proof does not carry through for arbitrary Plotkin orders. It is also unknown whether $|S|\left(T^{\omega}\right)$ is retraction universal for the strongly algebraic domains.

In [18] and [24] information systems are used to show how to solve recursive domain equations. Information systems are so representational that in some cases it is possible to get a solution up to equality (rather than just isomorphism). Since the convex powerdomain is not defined on information systcms, the method does not apply to the solution of equations involving it. However; there are some cases in which the category PLT can be used in this way. A short illustration can be given as follows. Suppose $P$ is a pre-order none of whose members are pairs and assume $\perp$ is an element which is not a pair and does not appear in $P$. We define a pre-order $(A, \vdash)$ which satisfies the equation $A=(P+S(A))_{\perp}$ as follows:

1. $\perp \in A$,

2. if $X \in P$ then $(\dot{X}, 0) \in A$,

3. if $u \subseteq A$ is finite then $(u, 1) \in A$,

4. if $X \vdash \perp$ for every $X \in A$,

5. $(X, 0) \vdash(Y, 0)$ if $X \vdash_{P} Y$,

6. $(u, 1) \vdash(v, 1)$ if $u \vdash s(A) v$.

It is easy to show that the poset $A$ defined in this way is a Plotkin order if $P$ is. 


\section{ACKNOWLEDGEMENTS.}

This research was carried out under the direction of Dana Scott with funding provided by Carnegie-Mellon University. The author thanks Jon Barwise for introducing him to Scott's work on domain theory. Thanks are also owed to Rick Statman and Gordon Plotkin for helpful conversations and correspondence. 


\section{REFERENCES}

[1] Bracho, F., Continuously Generated Fixed-Points. Doctoral Dissertation, Oxford University, 1983, $223 \mathrm{pp}$.

[2] Brookes, S., Semantics of Programming Languages. Pre-print, 1984, 200+ pp.

[3] Hrbacek, K., Powerdomain construction in the category of algebraic lattices. To appear in the proceedings of ICALP 85, Napflion, Greece. Lecture Notes in Computer Science, Springer-Verlag, 1985.

[4] Kamimura, T. and Tang, A., Finitely continuous posets. To appear in Theoretical Computer Science, 1985.

[5] Kanda, A., Fully effective solutions of recursive domain equations. In: MFCS 79, edited by J. Bečviar. Lecture Notes in Computer Science, vol. 74, Springer-Verlag, 1979.

[6] Kanda, A., Effective Solutions of Recursive Domain Equations. Doctoral Dissertation, Warwick University, 1980.

[7] Lambek J., From lambda-calculus to cartesian closed categories. In: To H. B. Curry: Essays on Combinatory Logic, Lambda Calculus and Formalism, edited by J. P. Seldin and R. Hindley. Academic Press, 1980, pp. 375 402.

[8] Lambek, J. and Scott, P. J., Introduction to Higher-Order Categorical Logic, Preprint, $1984,200+$ pp.

[9] MacQueen, D., Plotkin, G. D. and Sethi, R., An ideal model for recursive polymorhpic types. In: Eleventh Symposium on Principles of Programming Languages, edited by K. Kennedy, Association for Computing Machinery, 1984, pp. 165-174.

[10] Mulmuley, K., Full Abstraction and Semantic Equivalence. Doctoral Dissertation, Carnegie-Mellon University, 1985, 100+ pp.

[11] Plotkin, G. D., A powerdomain construction. SIAM Journal of Computing, vol. 5, 1976, pp. 452-487.

[12] Plotkin, G. D., The category of complete partial orders: a tool for making meanings. In: Proceedings of the Summer School on foundations of Artificial Intelligence and Computer Science, Instituto di Scienze dell'Informazione, University di Pisa, 1978.

[13] Plotkin, G. D., $T^{\omega}$ as a universal domain. Journal of Computer System Sciences, vol. 17, 1978, pp. 209-236.

[14] Scott, D. S., Continuous lattices. In: Toposes, Algebraic Geometry and Logic, edited by F. W. Lawvere. Lecture Notes in Mathematics, vol. 274, Springer-Verlag, 1972, pp. 97-136.

[15] Scott, D. S., Data types as lattices. SIAM Journal of Computing, vol. 5, 1976, pp. 522587. 
[16] Scott, D. S., Lectures on a mathematical theory of computation. Technical Report, no. PRG19, Oxford University Computing Laboratory, 1981, 148 pp.

[17] Scott, D. S., Some ordered sets in computer science. In: Ordered Sets, Banff, Canada, edited by I. Rival. D. Reidel Publishing Company, 1981, pp. 677-718.

[18] Scott, D. S., Domains for denotational semantics. In: ICALP 82, Aarhus, Denmark, edited by M. Nielsen and E. M. Schmidt. Lecture Notes in Computer Science, vol. 140, Springer-Verlag, 1982, pp. 577-613.

[19] Notes on cpo's/SFP-objects and the like. Manuscript, unpublished, 1982b, 14 pp.

[20] Smyth, M. B., Powerdomains. Journal of Computer System Sciences, vol. 16, 1978, pp. 23-36.

[21] Smyth, M. B., The largest cartesian closed category of domains. Theoretical Computer Science, vol. 27, 1983, pp 109-119.

[22] Smyth, M. B. and Plotkin, G. D., The category-theoretic solution of recursive domain equations. SIAM Journal of Computing, vol. 11, 1982, pp. 761-783.

[23] Stoy, J. E., Denotational Semantics: The Scott-Strachey Approach to Programming Language Theory. M.I.T. Press, 1977, 414 pp.

[24] Winskel, G. and Larsen, K., Using information systems to solve recursive domain equations effectively. In: Semantics of Data Types, Sophia-Antipolis, edited by G. Kahn and G. D. Plotkin. Lecture Notes in Computer Science, vol. 173, Springer-Verlag, 1984, pp. 109-130. 